\title{
Sensitivity of Tropical Cyclone Intensification to Axisymmetric Heat Sources: The Role of Low-Level Heating and Cooling from Different Microphysical Processes
}

\author{
Georgina Paull, ${ }^{a}$ Konstantinos Menelaou, and M. K. Yau \\ Department of Atmospheric and Oceanic Sciences, McGill University, Montreal, Quebec, Canada
}

(Manuscript received 24 January 2018, in final form 3 October 2018)

\begin{abstract}
Latent heat release from condensational heating has been recognized as one of the dominating energy sources of a tropical cyclone. Here we argue that other microphysical processes may also play an important role. From an analysis of a real-case simulation of Hurricane Katrina (2005), it was found that cooling from evaporation and melting of some frozen hydrometeors radially outside the eyewall region can have similar magnitudes as condensational heating. Based on this finding, idealized thermally forced experiments were performed. The specified heating and cooling functions mimic those found in the Hurricane Katrina run. The results indicated that the addition of cooling enhances the lower-level inward radial winds, which in turn increases the acceleration of the lower-level tangential winds through an enhanced transport of absolute vorticity. Sensitivity experiments on varying the structure of the cooling functions and the background state of the vortex demonstrate that the lower-level tangential wind acceleration is more sensitive to changes in the vertical structure and location of the cooling than the radial characteristics. In addition, the lower-level acceleration is sensitive to variations in the inertial and static stabilities rather than the vertical tangential wind shear of the initial vortex and its environment.
\end{abstract}

\section{Introduction}

Although tropical cyclone (TC) track forecast has greatly improved, such progress remains slow for the prediction of intensity (e.g., DeMaria and Mainelli 2005; Rogers et al. 2006, 2013). The multiscale nature of the processes responsible for intensity change is thought to be a significant reason for the relative lack of progress (Marks et al. 1998).

It is well documented that latent heating in the eyewall fueled by enthalpy fluxes from the ocean plays a major role in TC intensification (Malkus 1958; Malkus and Riehl 1960; Simpson and Halverson 1998; Willoughby 1998; Heymsfield et al. 2001; Zipser 2003). However, the role of ice processes and latent cooling is not as well understood. There is evidence that the inclusion of the ice phase in numerical simulations can lead to a very different TC structure when compared to simulations using only warm-rain microphysics (Lord 1984; Willoughby

\footnotetext{
${ }^{a}$ Current affiliation: Air Quality Research Division, Environment and Climate Change Canada, Dorval, Quebec, Canada.
}

Corresponding author: Georgina Paull, georgina.paull@mail. mcgill.ca et al. 1984). For instance, cooling from the melting of graupel may act to enhance the downdrafts and slow the vortex growth rate (Lord and Lord 1988). Despite the potential significance suggested from numerical studies, there has been limited direct observations of microphysical properties in TCs (Jin et al. 2014), especially direct observations of ice phase hydrometeors such as cloud ice and snow. Thus, Rogers (2010) stated that the lack of reliable TC intensity forecasts arises from the lack of understanding of the response of a TC vortex and its environment to the microphysical processes.

Over the last few decades, numerous studies using numerical models have been conducted to investigate the role of clouds and precipitation in affecting the intensity and vortex structure of TCs. These studies spanned a range of complexity, from idealized two-dimensional (2D) (or axisymmetric; e.g., Willoughby et al. 1984; Pendergrass and Willoughby 2009) and three-dimensional (3D) simulations (e.g., Fovell et al. 2009; Sawada and Iwasaki 2010) to realcase runs (e.g., Braun 2002; Zhu and Zhang 2006; Chan and Chan 2016). Notwithstanding this rich literature, modeling studies with a particular focus on how the ice phase may influence the dynamics of a TC are much more limited, and they tend to adopt a common strategy by performing sensitivity experiments in which some of the main 
microphysics source/sink terms (such as the terms regulating the melting of ice) are simply switched on and off (e.g., Zhu and Zhang 2006; Pattnaik and Krishnamurti 2007; Yamasaki 2013). Some common findings include 1) the vortex responds differently depending on whether the ice phase is included or only warm-rain microphysics are considered, and 2) the evolution of the vortex structure is sensitive to the specifics of a microphysics scheme. For example, McFarquhar et al. (2006) demonstrated that a TC can be sensitive to the prescribed fall speed of graupel particles. A similar conclusion is also reported by Fovell and $\mathrm{Su}$ (2007) in the context of a real-case study of Hurricane Rita (2005) and a number of idealized 3D experiments. Similarly, Sawada and Iwasaki (2007) investigated the sensitivity of a TC to various modifications within two specific microphysics schemes. They showed that the evolution of the TC and its structure are highly sensitive to cooling around the melting layer. Sawada and Iwasaki (2010) further found that when evaporative cooling is suppressed in a simulation, a stronger storm (in terms of maximum tangential winds and minimum surface pressure) results and the intensification is more rapid. Moreover, the radius of maximum wind and the radius of the outermost tangential winds become smaller. However, it should be mentioned that by suppressing various microphysical processes within an entire simulation, it is difficult to understand how cooling might contribute to the modification of the TC structure throughout different stages of its life cycle.

To summarize, previous research emphasized that TCs are highly sensitive to the microphysics (MP). In spite of this, exactly how each individual MP process modulates the characteristics of TCs is poorly understood. It is one of the main goals of the present study to make advances on this front during the intensification phase of a TC. In contrast to previous work that simply turns on or off some processes within an MP scheme followed by diagnosis of the resultant structural changes, we adopt here a different methodology. As a first step, a real-case simulation of Hurricane Katrina (2005) is performed. The heating and cooling terms resulting from each distinct microphysics process are then extracted and fitted with idealized heating/cooling functions. Simplified numerical experiments are then conducted in the context of an initially steady TC-like vortex thermally forced by these functions (individually or collectively) and the numerical results are analyzed. Thereafter, the generality of the findings is determined through a series of additional sensitivity experiments on varying various aspects of the simulation setup.

The remainder of this paper is organized as follows. Section 2 describes the computational setup for both the Hurricane Katrina (2005) and the accompanying idealized experiments. Section 3 provides an overview of the simulated Katrina and describes the major MP processes responsible for warming and cooling during the stage of intensification. Section 4 presents the results of the idealized runs. Section 5 forms the subject of additional sensitivity experiments. Section 6 contains a discussion and conclusions.

\section{Setup of the numerical experiments}

\section{a. Model configuration for the real Hurricane Katrina run}

The numerical simulation is conducted with the Weather Research and Forecasting (WRF) Model (Skamarock et al. 2008). The model configuration includes a $27-\mathrm{km}$ outer fixed domain and three two-waynested vortex-following inner domains with horizontal grid spacing of 9,3 and $1 \mathrm{~km}$, respectively. The vertical grid has 27 eta $(\eta)$ levels, with higher resolution in the planetary boundary layer, and the model top is located at $50 \mathrm{hPa}$. The initial and lateral boundary conditions (updated every $6 \mathrm{~h}$ ) are derived from the National Centers for Environmental Prediction (NCEP) Final operational global analysis data with $1^{\circ} \times 1^{\circ}$ spatial resolution. The simulation covers $72 \mathrm{~h}$, from 0000 UTC 27 August to 0000 UTC 30 August 2005.

A number of subgrid-scale parameterizations for various physical processes are used. Surface heat and moisture fluxes over water are provided by the Eta surface layer scheme based on the Monin-Obukhov similarity theory (Janjić 1996, 2002). Over land, these fluxes are computed with a simple five-layer thermal diffusion land surface scheme. The modified Mellor-Yamada-Janjić planetary boundary layer representation (Janjić 1994) is used to treat vertical turbulent mixing, whereas a Smagorinsky first-order closure is employed for horizontal turbulent mixing. Atmospheric longwave radiation is calculated using the Rapid Radiative Transfer Model for general circulation models (RRTMG) longwave (Iacono et al. 2008) scheme. The Goddard (Chou and Suarez 1999) scheme is used to compute shortwave radiation. In the outer two domains (27 and $9 \mathrm{~km}$ ), the Grell and Dévényi (2002) cumulus parameterization is used to simulate unresolved convection and precipitation. For microphysics (and also precipitation), the bulk Thompson et al. (2008) scheme is used, which explicitly predicts the mixing ratios of cloud water, rain, cloud ice, snow, and graupel.

\section{b. Model configuration for idealized experiments}

The idealized simulations employ a modified configuration of the WRF Model, intended to simplify the interpretation of the results. The fields evolve on an $f$-plane nonmoving single computational domain. The specified Coriolis parameter $f$ is $5 \times 10^{-5} \mathrm{~s}^{-1}$, which is representative of $20^{\circ} \mathrm{N}$. The horizontal domain spans $600 \mathrm{~km}$ in each orthogonal direction and the corresponding grid spacing 
is $2 \mathrm{~km}$. The vertical grid has 28 levels and extends upward to $z=25 \mathrm{~km}$, with $z$ denoting the height above sea level. At the upper boundary, a traditional Rayleigh damping layer is imposed above $z=20 \mathrm{~km}$ to prevent unphysical wave reflection from the upper boundary. Additionally, the vertical velocity is set to zero at the lower boundary, while the horizontal winds obey the free-slip condition. The lateral boundary conditions in both directions are periodic.

The initial conditions are specified by a stationary barotropic balanced axisymmetric TC-like vortex embedded in a quiescent flow with uniform sea surface temperature of $26^{\circ} \mathrm{C}$ (land is excluded). The radial profile of azimuthal wind in the initial vortex at $t=0$ follows that of Rotunno and Emanuel (1987) while the radius of maximum wind (RMW, located at $65 \mathrm{~km}$ ) and initial intensity $\left(50 \mathrm{~m} \mathrm{~s}^{-1}\right.$ ) closely resembles the magnitude of the simulated Katrina during its intensification stage at 1100 UTC 28 August. Specifically, the initial vortex is stationary due to a balance of forces, including gradient wind and hydrostatic balance. The corresponding pressure and density fields that keep the vortex in balance are obtained following an iterative process. Briefly, the far field vertical profiles of pressure and temperature are first prescribed from an initial sounding. The gradient wind equation is then integrated radially inward from the far field to obtain the two-dimensional (radius, height) pressure field. Note that, at this step, the temperature is held fixed to its current value. Following this, the hydrostatic balance is applied throughout the domain to correct both the temperature and the density. Finally, the previous two steps are repeated until the fields converge to a solution. Experiments (not shown) with the initial vortex without any thermal forcing reveal no rapid jump or rapid adjustment of both the flow and mass fields indicating that the initial vortex is highly balanced. We emphasize that the vortex is only barotropic at $t=0$. Thereafter, the vortex deviates from a barotropic vortex as it is driven by the thermal forcing in the WRF Model and is subject to the lower and upper boundary conditions.

All physical parameterization schemes are excluded. Motion is initially induced through a forcing term added in the prognostic equation for potential temperature. This "thermal" forcing is specified in accordance with the major MP processes diagnosed from the real Hurricane Katrina simulation. Specifically, each heating term and cooling term associated with a particular MP process is independently extracted and fitted to a function of the form:

$$
\begin{aligned}
\dot{\theta}(r, z, t)= & L(t) \times \dot{\theta}_{\max / \min } \exp \left[-\frac{\left(r-r_{c}-\gamma z\right)^{\alpha}}{\delta_{r}^{\alpha}}\right. \\
& \left.-\frac{\left(z-z_{c}\right)^{\beta}}{\delta_{z}^{\beta}}\right]
\end{aligned}
$$

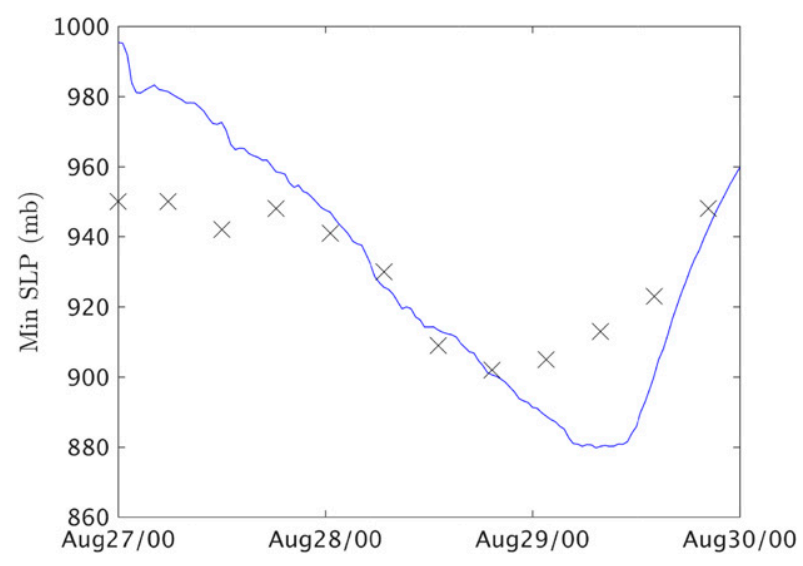

FIG. 1. Minimum SLP ( $\mathrm{mb} ; 1 \mathrm{mb}=1 \mathrm{hPa})$ for Hurricane Katrina from 27 to 30 August for the Thompson et al. (2008) microphysics scheme simulation (blue line) and the National Hurricane Center Data (crosses) from Knabb et al. (2005).

where $\dot{\theta}_{\max / \min }$ is the maximum amplitude of the heating or cooling rate, $r_{c}\left(z_{c}\right)$ is the center of the heating or cooling in the radial (vertical) direction, and $\delta_{r}\left(\delta_{z}\right)$ is the radial (vertical) half-width. The constant $\gamma$ determines the slope of the heating or cooling with height while the $\alpha$ and $\beta$ constants control the degree of decay in the radial and vertical directions, respectively. In some preliminary experiments it was found that when the full magnitude of the heating was turned on at time $t=0$, unphysical radiation of transient gravity waves from the heat source occurred. To reduce the radiation of these gravity waves due to the adjustment of the model, it was determined after some experimentation that the strength of the heating or cooling is best modulated by the $L(t)$ function which increased linearly with time from 0 to $1 \mathrm{~h}$ and then remained constant afterward. A similar procedure was used in Paull et al. (2017) with good results.

\section{Results of the real Hurricane Katrina simulation}

Figure 1 shows the time evolution of the simulated (blue line) and best track (black crosses) minimum sea level pressure (SLP). This is one baseline metric commonly used to gauge the performance of a real-case TC simulation. The simulation captures the intensification stage reasonably well, although it underestimates the initial intensity and overestimates the overall minimum pressure by approximately $20 \mathrm{hPa}$. The former discrepancy is primarily due to the coarse-resolution $\left(1^{\circ} \times 1^{\circ}\right)$ NCEP data used for the model initialization. The latter discrepancy might be the outcome of multiple reasons, with one hypothesis pointing toward inaccurate forecast of sea surface temperature (Zhu and Zhang 2006), while another hypothesis suggests this to be a result of weak model surface friction (Davis et al. 2008). Since our main 

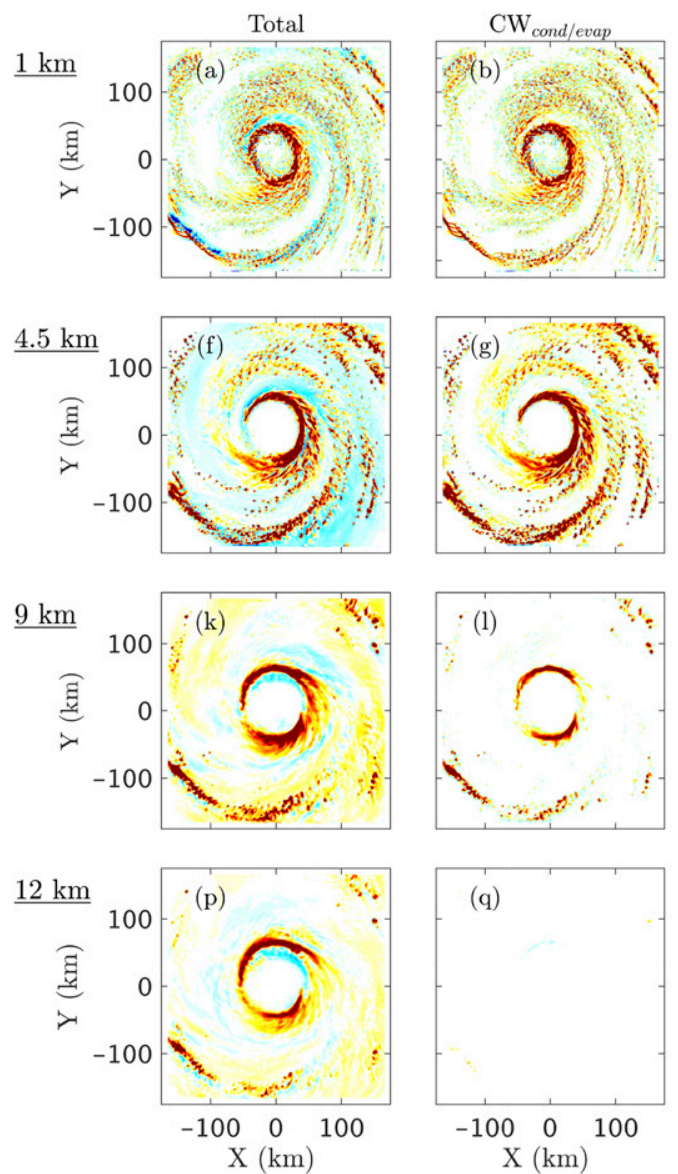

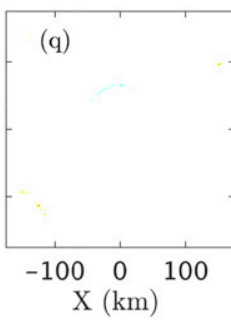

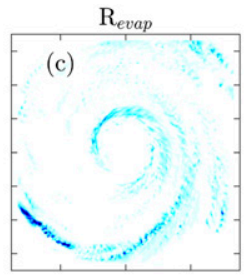

$(\mathrm{h})$

$\mathrm{S} / \mathrm{G}_{\text {melt }}$

(d)
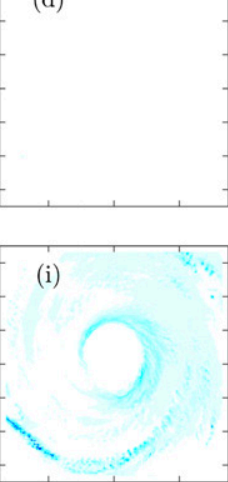

(n)
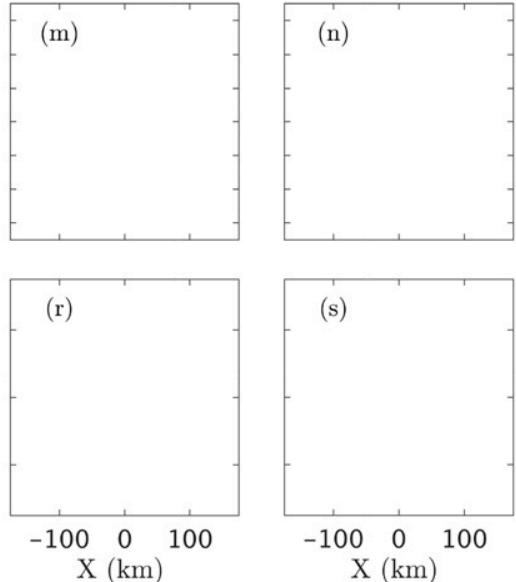

$\mathrm{X}(\mathrm{km})$
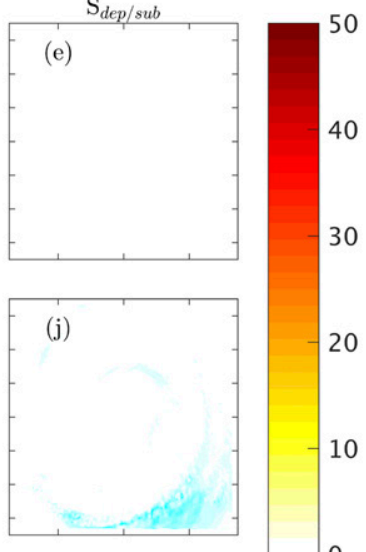

20

10

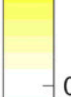

(o)
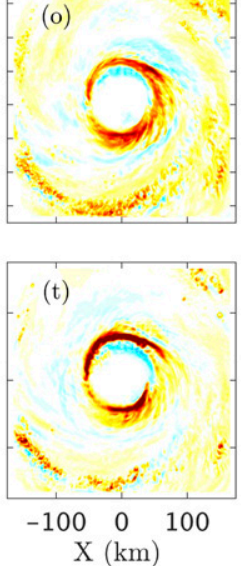

30

0

$-10$

$-20$

$-30$

$-40$

$-50$

$\mathrm{X}(\mathrm{km})$

FIG. 2. Horizontal cross sections at (a)-(e) 1-, (f)-(j) 4.5-, (k)-(o) 9-, and (p)-(t) 12-km height at 0500 UTC 28 August from the Hurricane Katrina simulation. (a),(f),(k),(p) The sum of all the diabatic processes $\left(\mathrm{K} \mathrm{h}^{-1}\right)$ at the given level. (b),(g),(l),(q) The heating (cooling) from the condensation (evaporation) of cloud water $\left(\mathrm{K} \mathrm{h}^{-1}\right)$. (c),(h),(m),(r) The cooling from the evaporation of rain $\left(\mathrm{K} \mathrm{h}^{-1}\right)$. (d),(i),(n),(s) The cooling from the melting of snow and graupel (e),(j),(o),(t) The heating (cooling) from the deposition (sublimation) of snow $\left(\mathrm{K} \mathrm{h}^{-1}\right)$. The red colors correspond to heating and the blue ones to cooling.

focus resides on the characteristics of the diabatic heating/cooling during intensification, an exact reproduction of Hurricane Katrina is not a necessity. A decomposition of the heating/cooling terms associated with all MP processes at two different times during the intensification phase is described below.

\section{Decomposition of diabatic MP processes}

Figures 2 and 3 show, respectively, the horizontal cross sections of the decomposed diabatic MP processes at four representative vertical levels at 0500 and 1100 UTC 28 August 2005. For reference, the total diabatic MP heating/cooling is displayed in the leftmost column. The condensation/evaporation of cloud water term is depicted in the second column, the evaporation of rainwater term in the third column, the melting of snow and graupel in the fourth column, and the sublimation/deposition of snow in the fifth column.
Each row represents a different height level: approximately above the boundary layer (at $1 \mathrm{~km})$, around the melting level of $0^{\circ} \mathrm{C}(4.5 \mathrm{~km})$, a level in the midtroposphere where both warm and cold MP processes are present $(9 \mathrm{~km})$, and a level in the upper troposphere where cold MP processes dominate $(12 \mathrm{~km})$.

Focusing first on the lowest level (top rows), it is clear that the majority of the heating in the vicinity of the eyewall results from the condensation of cloud water. Evaporation of cloud water is small as cloud water is mainly found in the eyewall and rainband regions which are saturated. Immediately outside of the positive heating region lies a ring of cooling due to rain evaporation (Figs. $2 \mathrm{c}$ and $3 \mathrm{c}$ ). This cooling is primarily the result of the evaporation of rain falling out of a tilted updraft into a subsaturated environment.

Around the melting level (second rows), the largest contributor to the net eyewall diabatic heating is again 

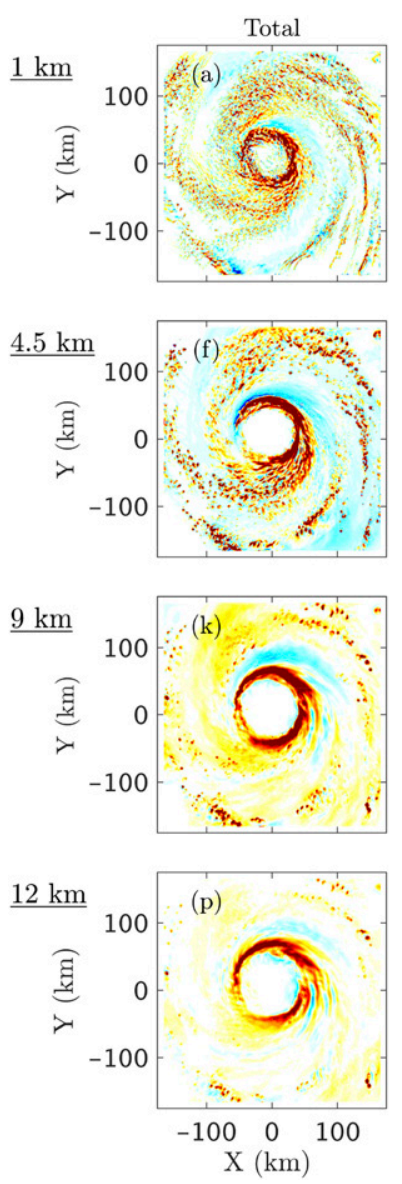
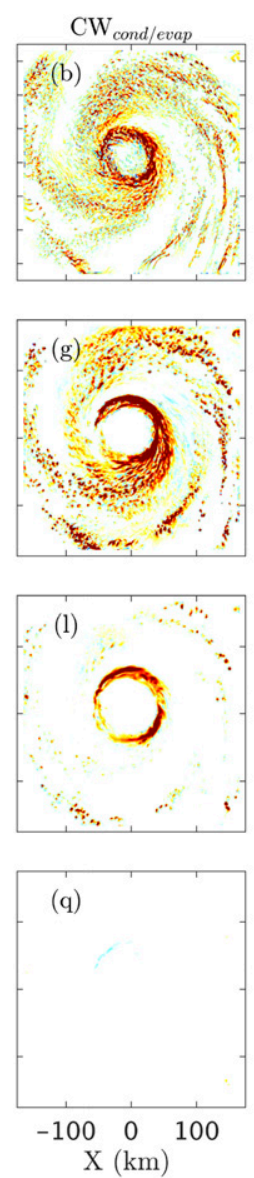
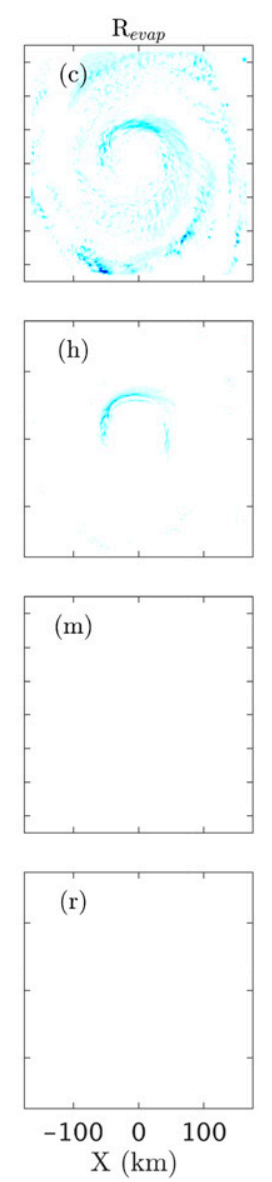
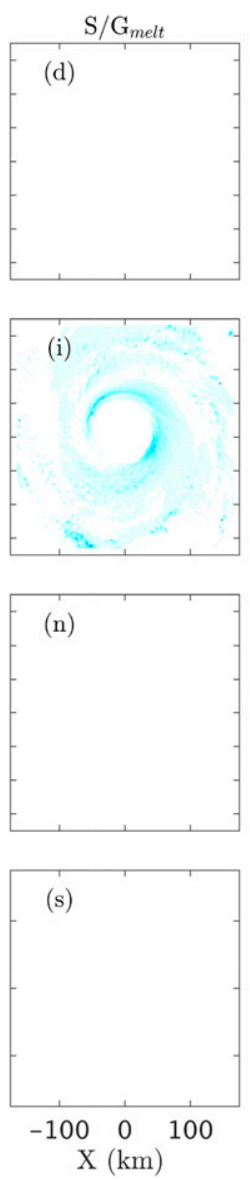
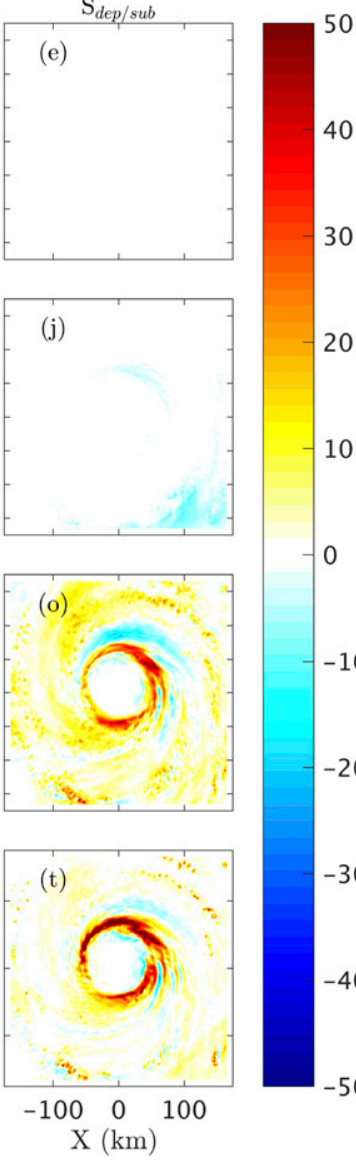

$-30$

20

10

0

FIG. 3. As in Fig. 2, but at 1100 UTC 28 Aug.

condensation of cloud water. Here, the highest positive values are approximately $60 \mathrm{~K} \mathrm{~h}^{-1}$. Directly adjacent to the area of heating, at a radius of about $55 \mathrm{~km}$, there exists a thin region of intense cooling with magnitudes on the order of $-30 \mathrm{~K} \mathrm{~h}^{-1}$ (addition of Figs. $2 \mathrm{~h}$ and $2 \mathrm{i}$ and of Figs. $3 \mathrm{~h}$ and 3i). The radial location of this intense cooling (outside the eyewall, produced by rain evaporation and the melting of graupel) is an indirect effect of an eyewall cloud that tilts radially outwards with increasing height. Moving to larger radii, there also exists a broad region of relatively weaker cooling (around $-15 \mathrm{Kh}^{-1}$ ) due to the melting of snow. The snow particles fall from the anvil region aloft and subsequently melt, giving rise to appreciable cooling of the surrounding environment.

It is of interest to note from a comparison of Figs. 2c and $2 \mathrm{i}$ (also Figs. $3 \mathrm{c}$ and $3 \mathrm{i}$ ) that the pattern of cooling from the melting of snow and graupel is very similar to the pattern of cooling from the evaporation of rain. On the other hand, the magnitude of evaporative cooling at $1 \mathrm{~km}$ is somewhat larger than the cooling by melting at $4.5 \mathrm{~km}$. These features imply that most of the rainwater at the lower levels originates from the melting of snow and graupel around the melting level, and the larger magnitude of evaporative cooling is attributable to the larger value of the latent heat of vaporization relative to the latent heat of fusion. In other words, ice hydrometeors (snow and graupel) contribute directly to latent cooling when they melt to become rainwater but also contribute indirectly to the "amplified" cooling at the lower levels when rainwater from the melted hydrometeors undergoes evaporation in an unsaturated environment. For this reason, the cooling from the melting of snow and graupel will be combined with the evaporative cooling of rainwater to study the impact on tangential wind acceleration in section 4 , as these processes mainly originate directly or indirectly from the same ice hydrometeor source.

At the midtropospheric level (third rows), condensation of cloud water and deposition of snow are clearly the two main heating processes, while cooling is predominantly due to the sublimation of snow. It should be mentioned that cloud ice is included in the Thompson et al. (2008) scheme. However, examination of the ice 
mixing ratio and snow mixing ratio at $9-14 \mathrm{~km}$ indicates that the value of the former is two orders of magnitude smaller than that of the latter. Consequently, cooling from cloud ice sublimation is negligible and is not plotted.

Within the eyewall at a height of $9 \mathrm{~km}$, the heating rate reaches $\sim 60 \mathrm{~K} \mathrm{~h}^{-1}$, similar to the magnitude at the melting level. On the other hand, cooling is much weaker (around $-10 \mathrm{Kh}^{-1}$ ) and is primarily distributed outside the eyewall. Some hints of snow sublimation are observed on the inner edge of the eyewall, which might enhance subsidence within the eye (Ohno and Satoh 2015).

Finally, at the upper level of the troposphere (fourth rows) where cold MP processes are expected to dominate, deposition and sublimation of snow accounts for most of the net diabatic heating and cooling, respectively, whereas condensation and evaporation of cloud water is quite negligible. Similar to the midtroposphere, the eyewall is characterized by large heating values, and only some weak cooling exists within its inner edge. In contrast to the midtroposphere, the magnitude of heating/cooling outside the eyewall region is much weaker.

The above decomposition indicates that during the stage of intensification, the eyewall region is characterized by consistent heating and cooling with comparable magnitudes. The decomposed patterns serve as a basis in the design of idealized 3D WRF simulations with the goal of obtaining a clearer understanding of how heating/ooling might influence low-level tangential wind acceleration.

\section{Sensitivity of low-level tangential wind intensification to different diabatic processes}

As shown in the previous section, condensation of cloud water and deposition of snow are the two main processes responsible for eyewall heating throughout the intensification stage. On the other hand, sublimation of snow, rain evaporation, and melting of snow and graupel tend to dominate the eyewall cooling. Using the results from Fig. 2 as a guide, we specify four thermal functions of the form given by Eq. (1) - one for condensation heating of cloud water, one for deposition heating of snow, one for sublimation cooling of snow, and one for the cooling from the combined effect of evaporation of rain and melting of snow and graupel. These functions (and a combination of them) are then used to force a series of idealized 3D simulations. The steps followed in specifying the four functions are explained below.

First, the heating/cooling terms induced by the major diabatic MP processes are output individually from the model. They are then azimuthally averaged and the variables $r_{c}$ and $z_{c}$ obtained from the radial and vertical locations of the maximum heating/cooling. The half widths $\delta_{r}$ and $\delta_{z}$ and the slope coefficient $\gamma$ are specified according to the structure of the simulated Hurricane Katrina. The exact values are listed in Table 1. Figure 4 compares the results from the specified functions (right column) with the azimuthally averaged structure from the simulated Katrina at 1100 UTC 28 August (left column). The first row represents a radius versus height cross sections of the cooling terms. The second row shows vertical cross sections taken through the vortex center of the total diabatic heating/cooling. It can be seen from Figs. $4 \mathrm{c}$ and $4 \mathrm{~d}$ that the sum of the four thermal functions compares reasonably well with the results from the simulated Katrina. As the simulated structures in Figs. 2 and 3 are quite similar, the specified heating/cooling functions are representative of the conditions during the intensifying stage, and not just at one specific time.

In total, eight idealized experiments are conducted. For discussion purposes, the diabatic processes are labeled according to their locations. Unless stated otherwise, we shall henceforth refer to the heating induced by condensation of cloud water as low-level heating (labeled LH), cooling induced by evaporation of rainwater and melting of snow and graupel as low-level cooling (labeled LC), heating induced by deposition of snow as upper-level heating (labeled UH), and cooling induced by the sublimation of snow as upper-level cooling (labeled UC). Table 2 lists the different combinations of thermal forcings in each experiment.

Because of the absence of friction and other physical processes in the idealized WRF experiments, applying the full strength of the thermal forcings listed in Table 1 would yield unrealistically large circulations in the lower levels. For this reason, in the idealized WRF runs the magnitudes of the thermal forcings in Table 1 are scaled down by a factor of 5. Specifically, the maximum heating rates and minimum cooling rates for $\mathrm{LH}, \mathrm{LC}, \mathrm{UH}$, and $\mathrm{UC}$ are reduced from $50,-15,25$, and $-5 \mathrm{~K} \mathrm{~h}^{-1}$ to $10,-3,5$, and $-1 \mathrm{Kh}^{-1}$, respectively. The other parameters in Table 1 remain unchanged.

Figure 5 depicts the time evolution of the resultant low-level tangential wind acceleration. During the first hour, all cases registered a gradual increase in acceleration as the forcing is specified to rise linearly to its maximum value over the 1-h period. After the first hour, the results tend to fall into two distinct branches. The first branch (made up of the blue curves) comprises all cases that include LC. The second branch (made up of the red curves) consists of all cases that exclude LC. It can be seen that whenever LC is included (excluded), the simulations exhibit relatively larger (smaller) wind accelerations. 
TABLE 1. Constants used in Eq. (1).

\begin{tabular}{|c|c|c|c|c|c|c|c|c|c|}
\hline MP process & $\begin{array}{c}\text { Max from } \\
\text { Katrina }\left(\mathrm{K} \mathrm{h}^{-1}\right)\end{array}$ & $\dot{\theta}_{\max / \min }\left(\mathrm{K} \mathrm{h}^{-1}\right)$ & $r_{c}(\mathrm{~km})$ & $\delta_{r}(\mathrm{~km})$ & $\gamma$ & $z_{c}(\mathrm{~km})$ & $\delta_{z}(\mathrm{~km})$ & $\alpha$ & $\beta$ \\
\hline $\mathrm{LH}$ & 60 & 50 & 43 & 10 & 1 & 5.5 & 4 & 2 & 2 \\
\hline $\mathrm{LC}$ & -30 & -15 & 60 & 10 & 1 & 3.5 & 2 & 2 & 2 \\
\hline $\mathrm{UH}$ & 60 & 25 & 54 & 8 & 0 & 10.5 & 2 & 2 & 2 \\
\hline $\mathrm{UC}$ & -10 & -5 & 42 & 4 & 0 & 11 & 2 & 2 & 2 \\
\hline
\end{tabular}

Within each branch, there are subtle differences as depicted in the magnified inset (right panel of Fig. 5) of the normalized acceleration from 100 to $135 \mathrm{~min}$. The normalization is performed with respect to the LH only run. Note that when UH is included (excluded), the lowlevel wind acceleration becomes stronger (weaker). The role of the upper-level heating in causing these subtle differences will be addressed in a future publication.

Returning to the question of why the presence of LC leads to a larger acceleration, we plotted in Fig. 6 the azimuthally averaged tangential wind acceleration and secondary circulation for experiments LH (Fig. 6a) and LH + LC (Fig. 6b) at 180 min of simulation time. For $\mathrm{LH}$, the structure of the mean secondary circulation resembles the classic form, with a low-level radial inflow, a corresponding vertical ascent through the heat source, and a radial outflow above the heating maximum. On the other hand, for LH + LC the structure is somewhat different. Here, the presence of LC induces vertical descent through its center, which in turn acts to enhance the lowlevel circulation produced by LH. In other words, there seems to exist a coupling between $\mathrm{LH}$ and LC that results in a stronger low-level radial inflow. Stronger inflow implies larger radial transport of low-level absolute angular momentum, which further produces larger acceleration (as evident by the plotted magnitudes).

A question may arise as to why the cooling located outside the RMW with $1 / 3$ of the magnitude of the heating can have such a strong impact? Pendergrass and Willoughby (2009) demonstrated when the heating was
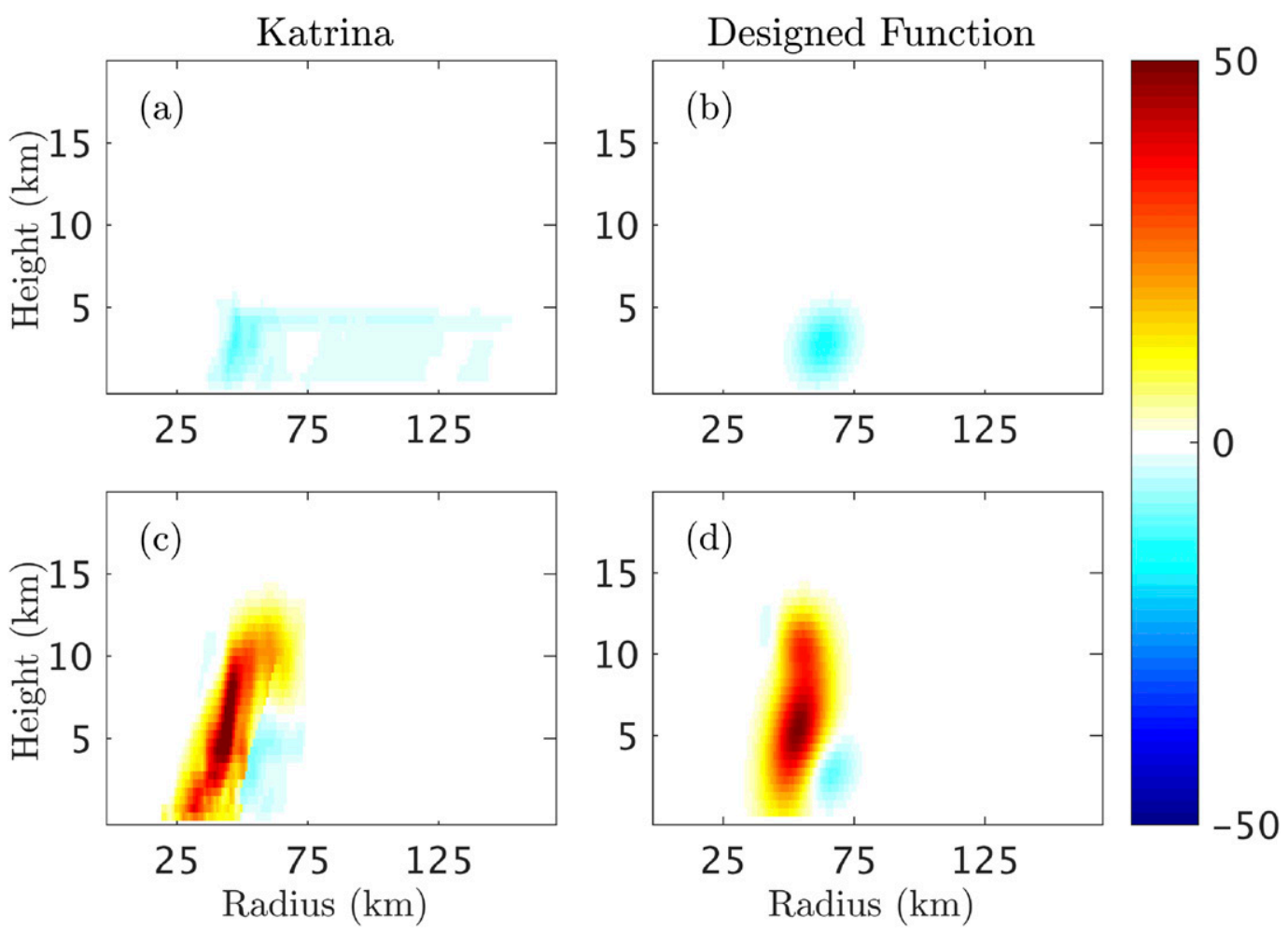

FIG. 4. Radius vs height examples of azimuthally averaged MP processes from (left) Hurricane Katrina and (right) the respective process fitted to Eq. (1) $\left(\mathrm{K} \mathrm{h}^{-1}\right)$. (a),(b) The cooling processes associated with the evaporation of rainwater and melting of ice hydrometeors. (c),(d) The total diabatic heating/cooling profile of Katrina and the designed function $\left(\mathrm{K} \mathrm{h}^{-1}\right)$, respectively. 
TABLE 2. Description of the lines in Fig. 5.

\begin{tabular}{cll}
\hline \hline Simulation & Line description & MP processes included \\
\hline 1 & Red solid & LH \\
2 & Red dotted & LH and UC \\
3 & Red dash-dotted & LH and UH \\
4 & Red dashed & LH, UC, and UH \\
5 & Blue solid & LH and LC \\
6 & Blue dotted & LH, UC, and LC \\
7 & Blue dashed & LH, LC, and UH \\
8 & Blue dash-dotted & LH, LC, UH, and UC \\
\hline
\end{tabular}

located in an area of lower inertial stability, such as the region located outside the RMW, there is a weaker induction of the secondary circulation and, thus, a weaker acceleration of the low-level tangential winds. To answer this question, it should first be remembered that in a strictly balanced scenario [using, for instance, the diagnostic Sawyer-Eliassen (SE) equation similar to Pendergrass and Willoughby (2009)] the magnitude (and structure) of the flow induced by a prescribed heat source is largely determined by the radial and vertical gradients of the heat source and not by its magnitude. This can be clearly seen by examining the RHS of Eq. (9) in Pendergrass and Willoughby (2009). As such, even a small additional cooling outside of the heating may result in large differences in the overall radial gradient of the source term within a balanced diagnostic model to produce a large impact.

To further substantiate the above finding, we performed three experiments (SE1, SE2, and SE3, which are further explained in the appendix) using the SE equation to assess whether in this balanced framework the presence of LC can lead to a larger low-level acceleration demonstrated in the idealized WRF simulations. In SE1, the thermal forcing consists only of LH. In SE2, both LH and LC are included. In SE3, the SE equation is again forced with both $\mathrm{LH}+\mathrm{LC}$ but with a sharper radial decay of LC (controlled by the parameter $\alpha$, with $\alpha=4)$. The plots of LH and LH + LC (refer to Figs. A1 and $\mathrm{A} 2$ in the appendix) indicate that the addition of even weak outside cooling ( $1 / 3$ the magnitude of inside heating) can result in significant differences in the magnitude of the forcing terms in the SE model (especially the one related to the radial gradient of the thermal forcing). Furthermore, the patterns of the low-level tangential wind acceleration (not shown) are qualitatively similar to those from the idealized WRF simulations. There are some quantitative differences due largely to the fact that the vertical advection of azimuthal momentum is zero in the SE framework but is nonzero in the prognostic WRF framework. Additionally, the largest acceleration occurs in the presence of cooling. Moreover, when LH in SE1 and LH + LC in SE2 are moved radially inward toward regions of higher inertial stability, the maximum acceleration becomes stronger particularly for the LH + LC case (refer to Fig. A3 in the appendix), implying that the conclusion of the enhanced circulation with outside cooling remains valid for different values of the inertial stability at the forcing location. The consistency of the findings from the SE model and the idealized WRF runs provides further confidence that the presence of relatively weak outside cooling can indeed induce nontrivial changes in the acceleration of the low-level winds.
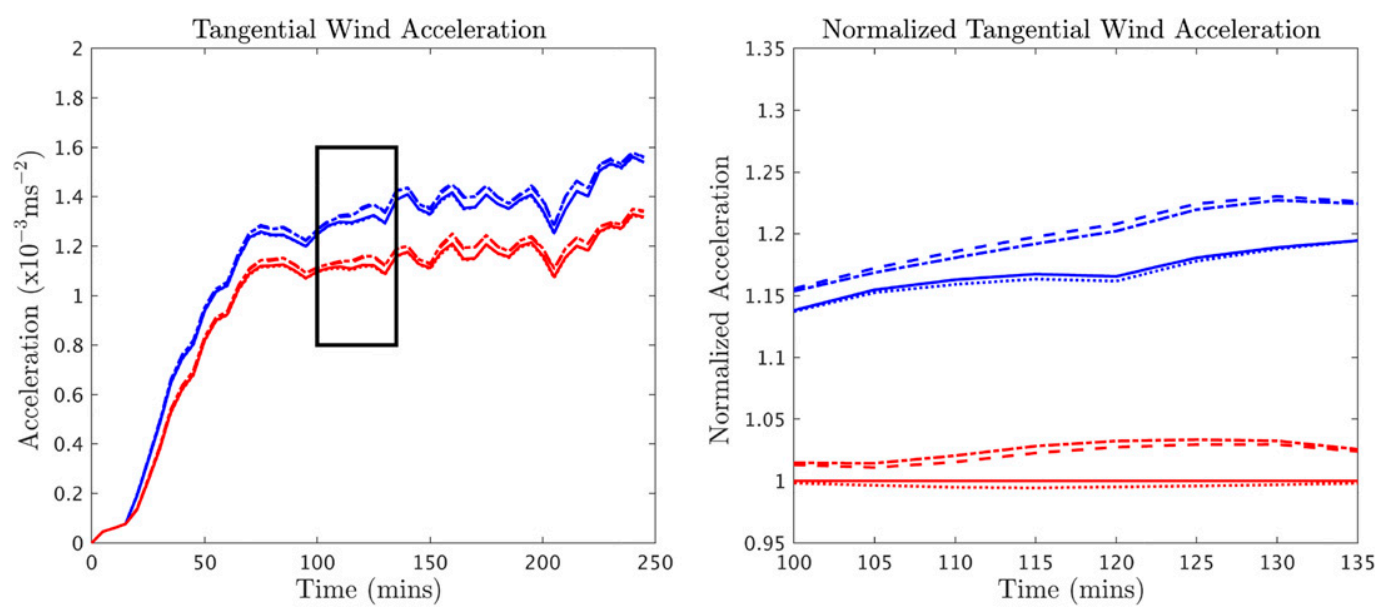

FIG. 5. (left) Time (min) vs tangential wind acceleration $\left(\times 10^{-3} \mathrm{~m} \mathrm{~s}^{-2}\right)$ for the eight different simulations outlined in Table 2 . The blue curves indicate that the LC is included in the simulation, whereas the red curves indicate that the LC is excluded. Refer to Table 2 for the description of all of the lines. The box is an outline of the expanded section from 100 to $135 \mathrm{~min}$. (right) Expanded time vs normalized tangential wind acceleration plot based on the box in the left panel. The normalization is taken with respect to the LH-only run. 
Tang. Wind Acc. for LH Only

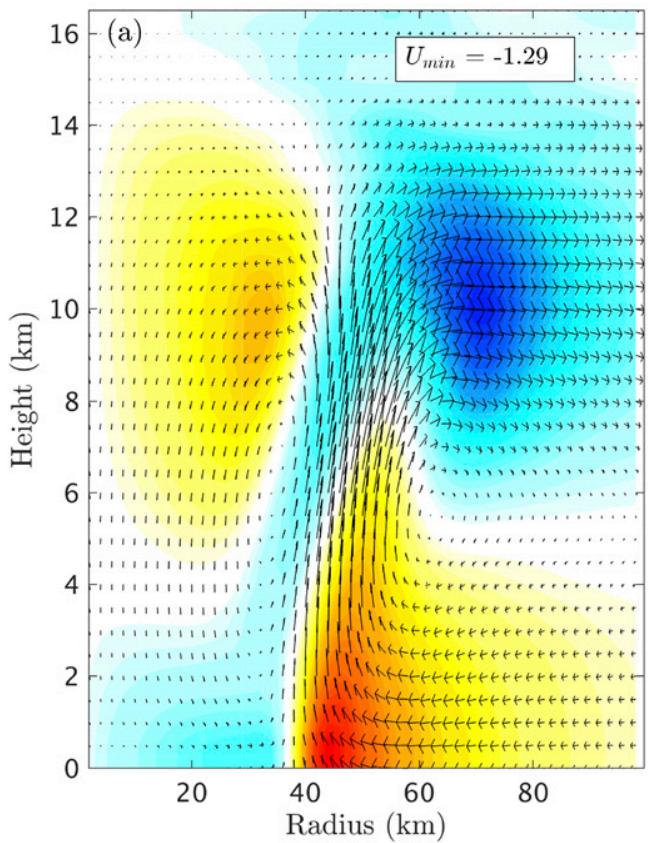

Tang. Wind Acc. for $\mathrm{LH}$ and LC

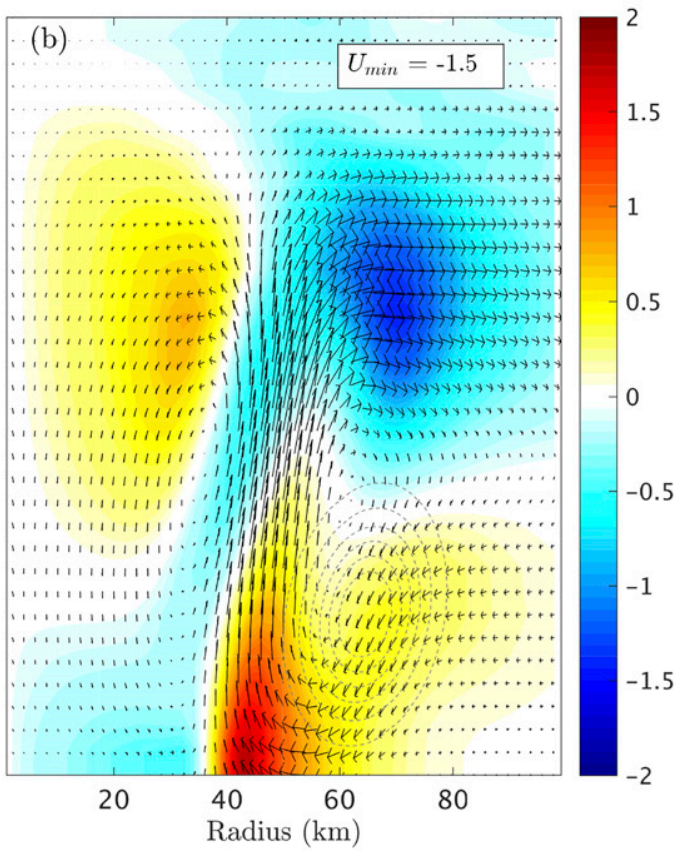

FIG. 6. The azimuthally averaged radius vs height cross section of the tangential wind acceleration $\left(\times 10^{-3} \mathrm{~m} \mathrm{~s}^{-2}\right)$ calculated at $180 \mathrm{~min}$ for (a) LH only and (b) LH + LC simulation. The red colors represent an acceleration of the tangential winds and the blue colors denote a deceleration. The arrows represent the induced secondary circulation. Lightly dashed contours in the background of (b) represent an outline of the LC.

Another question that naturally arises is whether this coupling is restricted to the particular configuration of the numerical experiment or whether it is a genuine feature. To address this question, we will provide in the following section the results from additional sensitivity experiments on varying the structures of the background vortex, the embedded environment, and the thermal forcing.

\section{Additional sensitivity experiments}

This section concentrates on two sets of sensitivity experiments. One set focuses on the modification of the thermal forcing, specifically on varying the main parameters that control the structure of the LC forcing [refer to Eq. (1)] while keeping LH unchanged. The second set represents changes to the structure of the background vortex and its environment. The design of the second set is motivated by the fact that in a balanced framework, the response of a TC to internal forcing is dependent on the structural parameters of inertial stability, static stability, and baroclinicity (Holland and Merrill 1984). Thus, these three parameters will be varied.

To assess the response of the acceleration, a decomposition of the azimuthally averaged tangential wind equation is performed as follows:

$$
\frac{\partial \bar{v}}{\partial t}=-\bar{u}(\overline{\zeta+f})-\bar{w} \frac{\overline{\partial v}}{\partial z}-\overline{u^{\prime}} \overline{\zeta^{\prime}}-\overline{w^{\prime}} \frac{\overline{\partial v^{\prime}}}{\partial z}
$$

where all the variables are azimuthally averaged and have their normal meanings. On the RHS of Eq. (2) the first and third terms together represent the total radial flux of absolute vorticity, and the second and fourth terms are the vertical advection of tangential momentum by the mean flow and eddies, respectively. Our analysis emphasizes the acceleration at the lower levels governed principally by the total radial flux of absolute vorticity. As was shown in Paull et al. (2017), the contribution from the vertical advection of tangential momentum only becomes significant at higher levels.

\section{a. Modification of the LC forcing}

Before proceeding, it is worth recalling that LC incorporates the cooling induced by rain evaporation and melting of snow and graupel. With this in mind, it is reasonable to expect that its overall structure (and characteristics relative to $\mathrm{LH}$ ) is not restricted to the particular setup and might vary for different TCs. For example, the relative radial distance of $\mathrm{LC}$ to $\mathrm{LH}$ can differ when two TCs are characterized by eyewalls that tilt differently with height. Also, the relative vertical 
TABLE 3. Description of sensitivity experiments to Eq. (1).

\begin{tabular}{cl}
\hline \hline Parameter of Eq. (1) & \multicolumn{1}{c}{ Values used } \\
\hline$r_{c}$ & $55,65,75,85 \mathrm{~km}$ \\
$z_{c}$ & $3,4,4.5,5 \mathrm{~km}$ \\
$\delta_{r}$ & $5,7,13,15 \mathrm{~km}$ \\
$\delta_{z}$ & $1,1.5,2.5,3 \mathrm{~km}$ \\
$\alpha$ & $1.6,1.8,2.2,2.4$ \\
$\beta$ & $1.5,2.5,3,4$ \\
\hline
\end{tabular}

distance of LC to LH may differ for TCs with different melting levels. Here, the potential impact of such variations to the aforementioned coupling hypothesis is investigated by modifying systematically the parameters $r_{c}, z_{c}, \delta_{r}, \delta_{z}, \alpha$, and $\beta$ in Eq. (1) as listed in Table 3.

Figure 7 contains a summary of the results by depicting the time evolution of the tangential wind acceleration in the time span from 60 to $250 \mathrm{~min}$. Each panel illustrates the sensitivity to variation of one particular parameter given in the subtitle. The remaining parameters take on the default value listed in Table 1 . The different blue curves correspond to different values, with dashed being the smallest, dotted being the largest, and dash-dotted and solid the values in between. The red curve indicates the resultant wind acceleration when only LH is considered (provided here as a reference).

The significance of the relative central distance (radially and vertically) between LC and LH is reflected in Figs. $7 \mathrm{a}$ and $7 \mathrm{~b}$. In general, the closer (farther) LC is to $\mathrm{LH}$, the larger (smaller) is the low-level acceleration of the tangential winds, consistent with the change in the radial gradient of the forcing discussed previously. This result also appears to be a direct outcome of the coupling hypothesis, with the degree of coupling inversely proportional to the separation distance of the two opposite
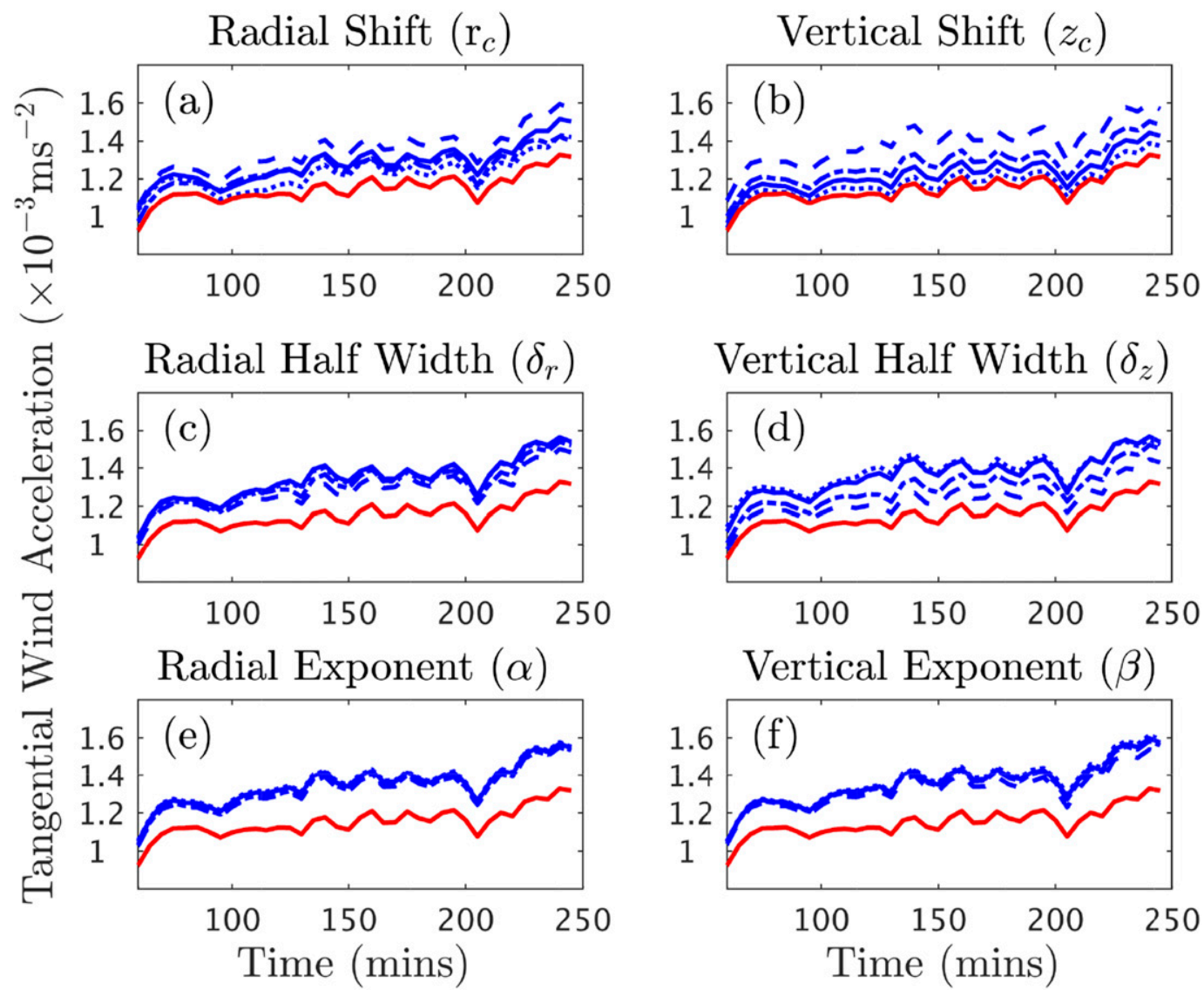

FIG. 7. Time ( $\mathrm{min})$ vs tangential wind acceleration $\left(\times 10^{-3} \mathrm{~m} \mathrm{~s}^{-2}\right)$ at the lower level for six different sensitivity tests to the constants from Eq. (1). The solid red lines in all panels represent the LH-only run. The different blue curves correspond to different values, with the smallest value being represented by a dashed line, followed by a dash-dotted line, then a solid line, and the largest is a dotted line. The panels are sensitivity tests to (a) the radial location of the cooling where $r_{c}=55,65,75$, and $85 \mathrm{~km},(\mathrm{~b})$ the vertical location of the cooling where $z_{c}=3,4,4.5$, and $5 \mathrm{~km}$, (c) the radial half-width where $\delta_{r}=5,7,13$, and $15 \mathrm{~km}$, (d) the vertical half-width where $\delta_{z}=1,1.5,2.5$, and $3 \mathrm{~km}$, (e) the radial exponent where $\alpha=1.6,1.8,2.2$, and 2.4, and (f) the vertical exponent where $\beta=1.5,2.5,3$, and 4 . 
magnitude sources. A stronger coupling implies a more intense radial inflow which in turn induces larger inward transport of absolute angular momentum to accelerate the tangential wind. Additionally, Fig. $7 \mathrm{~b}$ indicates that the lower the vertical location of the cooling, the stronger the tangential wind acceleration becomes. The melting of snow and graupel to form rainwater, which in turn evaporates at the lower levels, shifts the vertical location of the cooling downward. This effect, coupled with the enhanced cooling due to a phase change from ice to water, would further accelerate the low-level tangential wind.

The influence of the shape characteristics is depicted in Figs. 7c-f. The results indicate that the acceleration is more sensitive to changes induced in the vertical extent of LC (Fig. 7d), and less sensitive to the remaining parameters considered. Despite this, a general key outcome is that in all the plots the red curve (LH only case) has systematically the smallest values, indicating that the presence of LC leads to more intense storms than the case without.

\section{b. Modifications to the vortex parameters}

Here we modify the initial background parameters of the vortex in terms of the inertial stability, baroclinicity, and static stability. The details of the modification are given in Table 4.

\section{1) INERTIAL STABILITY}

The changes in inertial stability follow Paull et al. (2017). The definition of inertial stability is given by

$$
I^{2}=(\zeta+f)\left(\frac{2 v}{r}+f\right)
$$

where all the variables have their normal meanings. This parameter $I^{2}$ is generally considered as a metric for the resistance to movement of air parcels in the radial direction: the higher the inertial stability, the stronger the resistance to radial movement. Therefore, if a given heat source lies in a high-inertial-stability region, a reduction in the forced secondary circulation (radial and vertical winds) is expected (e.g., Hack and Schubert 1986; Pendergrass and Willoughby 2009; Paull et al. 2017).

In total, nine pairs of sensitivity simulations are performed. Each pair consists of an $\mathrm{LH}+\mathrm{LC}$ run and an LH only run. The runs are all initialized with a basicstate vortex with the same spatial structure $\left(r_{\max }=\right.$ $55 \mathrm{~km}$ ) but different maximum azimuthal wind $v_{\max }$. The maximum speed ranges from 15 to $55 \mathrm{~m} \mathrm{~s}^{-1}$ with $5 \mathrm{~m} \mathrm{~s}^{-1}$ increments and spans $I^{2}=[0.21,2.87] \times 10^{-5} \mathrm{~s}^{-2}$. The different $v_{\text {max }}$ values cover that of a tropical depression to a category $3 \mathrm{TC}$.

Despite nine pairs of simulations being conducted, for clarity of presentation, Fig. 8a shows only two pairs of
TABLE 4. Description of sensitivity experiments to the background vortex.

\begin{tabular}{cc}
\hline \hline Background vortex parameter & \multicolumn{1}{c}{ Values used } \\
\hline$I_{\max }^{2}$ & {$[0.21,2.87] \times 10^{-5} \mathrm{~s}^{-2}$} \\
$B^{2}$ & $25 \%, 50 \%, 75 \%, 100 \%$ \\
$N^{2}(6.5 \mathrm{~km})$ & $(1.07,1.32,2.20) \times 10^{-4} \mathrm{~s}^{-2}$ \\
\hline
\end{tabular}

simulations, one for the weakest vortex (green lines) and the other for the strongest vortex (black lines). Here the solid curves correspond to the runs with $\mathrm{LH}+\mathrm{LC}$ while the dashed curves are for the experiments with LH only. For the strongest vortex, there is a clear enhancement of the lower-level tangential wind acceleration when LC is present. For the weakest vortex, the enhancement is less conspicuous but there is a clear signature after $150 \mathrm{~min}$ of run time. Additionally, the tangential wind acceleration for the strongest vortex is larger than the weakest vortex, despite a larger $I^{2}$ and hence stronger resistance to the radial inflow. The reason for this behavior can be traced to the larger initial absolute vorticity prescribed in the stronger vortices. As a result, the lower-level radial flux of absolute vorticity becomes greater for a strong vortex despite a weaker secondary circulation, consistent with the finding reported in Paull et al. (2017). Further support for this view can be obtained through an analysis of the tangential wind equation [Eq. (2)] by computing the first term on its RHS.

Specifically, at the radial location of the maximum tangential wind acceleration for each of the two pairs of experiments, the azimuthal and temporal averages of the absolute vorticity and radial winds between 60 and $250 \mathrm{~min}$ were calculated. For the LH only cases, the averaged radial winds were $-0.83(-0.54) \mathrm{m} \mathrm{s}^{-1}$ for the weaker (stronger) vortex. Similarly, for the LH + LC simulations, they were $-0.68(-0.66) \mathrm{ms}^{-1}$. The corresponding averaged absolute vorticity in the $\mathrm{LH}$ and $\mathrm{LH}+\mathrm{LC}$ runs were 0.62 $(1.9) \times 10^{-3}$ and $0.91(1.8) \times 10^{-3} \mathrm{~s}^{-1}$ for the weaker (stronger) vortex, respectively. Multiplying these values together with a minus sign in front [the first term on RHS of Eq. (2)], the contribution of the radial flux of absolute vorticity to the lower-level tangential wind acceleration for the weaker (stronger) vortex turns out to be $\partial v / \partial t=$ $0.53(1.0) \times 10^{-3} \mathrm{~s}^{-2}$ for the LH only runs and $\partial v / \partial t=$ $0.62(1.2) \times 10^{-3} \mathrm{~s}^{-2}$ for the $\mathrm{LH}+\mathrm{LC}$ experiments.

It is clear that in both the weaker and stronger vortices there is an enhancement of the acceleration of the tangential winds when LC is included. This enhancement is a result of the increased inward radial transport of absolute vorticity. The response of the stronger vortex is larger than the weaker vortex, implying that the enhancement from the cooling is more effective for a more intense storm. 
Inertial Stability $\left(\mathrm{I}^{2}\right)$

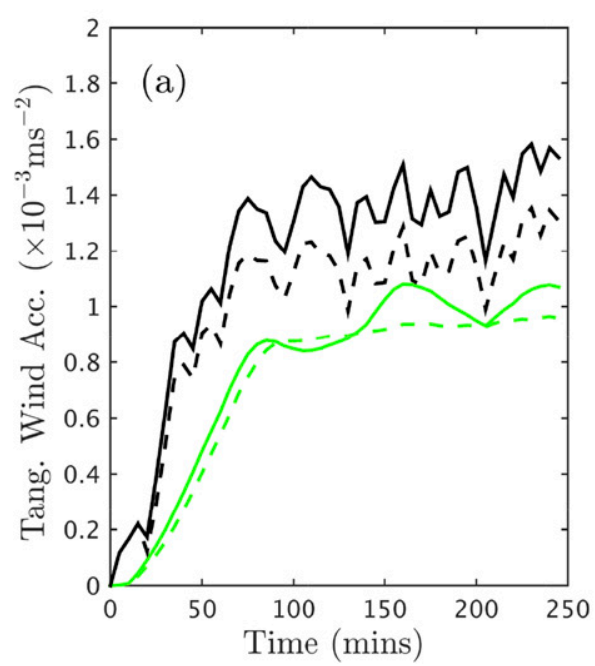

Baroclinicity $\left(\mathrm{B}^{2}\right)$

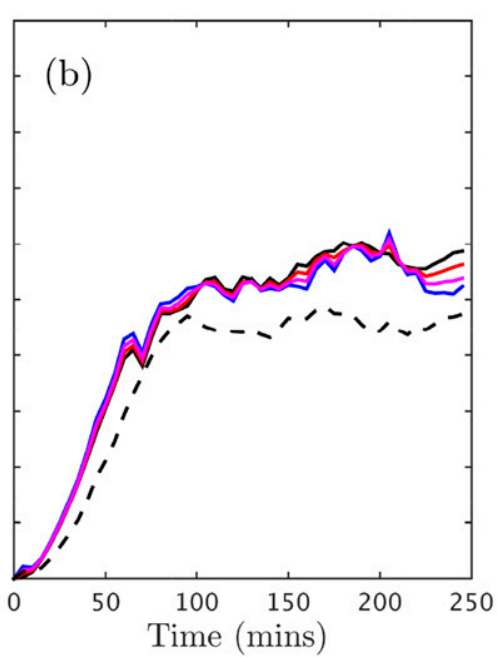

Static Stability $\left(\mathrm{N}^{2}\right)$

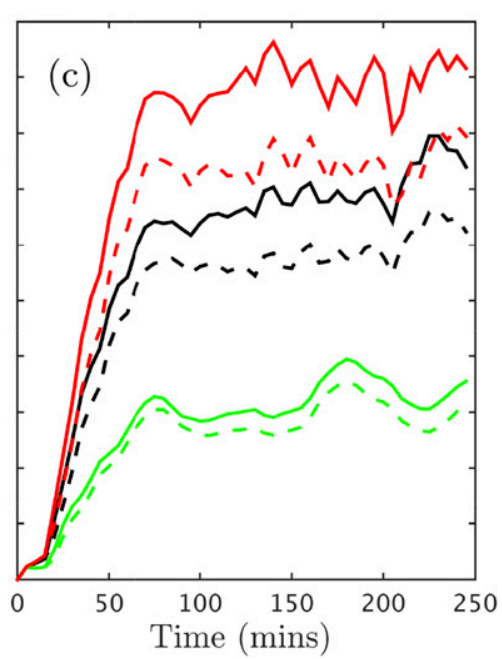

FIG. 8. Time ( $\mathrm{min})$ vs tangential wind acceleration $\left(\times 10^{-3} \mathrm{~m} \mathrm{~s}^{-2}\right)$ at the lower level of the LH-only (dashed lines) and LH $+\mathrm{LC}($ solid lines) simulations for (a) inertial stability $I^{2}$, (b) baroclinicity $B^{2}$, and (c) static stability $N^{2}$. The inertial stability shows the weakest vortex $\left(I_{\max }^{2}=0.21 \times\right.$ $10^{-5} \mathrm{~s}^{-2}$ in green) and the strongest vortex $\left(I_{\max }^{2}=2.87 \times 10^{-5} \mathrm{~s}^{-2}\right.$ in black). The different values of baroclinicity are $25 \%$ (magenta), $50 \%$ (red), $75 \%$ (blue), and $100 \%$ (black). The simulations for static stability are the doubling of the static stability $\left[N^{2}(6.5 \mathrm{~km})=2.2 \times 10^{-4} \mathrm{~s}^{-2}\right.$ in green], reversing bottom $6 \mathrm{~km}\left[N^{2}(6.5 \mathrm{~km})=1.07 \times 10^{-4} \mathrm{~s}^{-2}\right.$ in red $]$ and constant static stability $\left[N^{2}(6.5 \mathrm{~km})=1.32 \times 10^{-4} \mathrm{~s}^{-2}\right.$ in black].

\section{2) BAROCLINICITY}

For the sensitivity on baroclinicity, four different vortices are constructed, referred to respectively as the $25 \%, 50 \%, 75 \%$, and $100 \%$ baroclinic experiments. The degree of baroclinicity is governed by the vertical tangential wind shear $\partial v / \partial z$ obtained from a linear combination of a pure barotropic vortex and a baroclinic vortex given in Rotunno and Emanuel (1987). Specifically, the tangential winds in the $25 \%$ baroclinic case are calculated by adding $25 \%$ of the tangential winds from the baroclinic vortex to $75 \%$ of the tangential winds from the barotropic vortex. The specification of the $50 \%$ and $75 \%$ baroclinic cases followed a similar procedure. The winds in the $100 \%$ baroclinic experiment are simply given by the Rotunno and Emanuel (1987) vortex. Once the initial winds are specified, an iterative procedure between the temperature field and the tangential wind field is carried out to ensure approximate thermal and gradient wind balance (Rotunno and Emanuel 1987).

The solid lines in Fig. $8 \mathrm{~b}$ correspond to the simulations with different degrees of baroclinicity when both $\mathrm{LH}$ and LC are included. The dashed line represents the LH-only run for the $25 \%$ baroclinic experiment (the curves for the $50 \%, 75 \%$, and $100 \%$ baroclinicity LHonly simulations are all similar and therefore not shown). Two features can be detected. First, for all runs with different degrees of baroclinicity, the LH-only experiments always indicate weaker tangential wind acceleration relative to the $\mathrm{LH}+\mathrm{LC}$ experiments. Second, the low-level wind acceleration seems insensitive to the degree of baroclinicity specified. This latter result can be explained with the aid of Eq. (2). The tangential wind acceleration at the lower levels is mainly governed by the radial flux of absolute vorticity. Since the degree of baroclinicity has little impact on the lower-level maximum radial winds or the maximum absolute vorticity, low-level tangential wind acceleration is not much affected. However, differences in baroclinicity do influence the vertical structure of the tangential wind acceleration through the second and fourth terms on the RHS of Eq. (2). A larger degree of baroclinicity yields larger tangential wind acceleration higher up where the vertical winds are stronger (not shown). Consequently, the vertical advection of tangential wind momentum increases. The effect of the increase in vertical tangential wind momentum transport is outside the scope of the present paper and will be reported in a future publication.

\section{3) Static STABility}

The static stability profiles are altered by changing the potential temperature $\theta$ in the original input sounding with a corresponding change in the Brunt-Väisälä frequency:

$$
N^{2}=\frac{g}{\theta} \frac{\partial \theta}{\partial z}
$$


Three profiles of $N^{2}$ are investigated. The first one represents an atmosphere with a constant static stability $\left[N^{2}(6.5 \mathrm{~km})=1.32 \times 10^{-4} \mathrm{~s}^{-2} \text {; black curves in Fig. } 8 \mathrm{c}\right]^{1}$ from the surface to a height of approximately $12 \mathrm{~km}$ and then becomes significantly more stable higher up. The second profile represents a doubling of the original static stability [from Jordan (1958); $N^{2}(6.5 \mathrm{~km})=2.2 \times$ $10^{-4} \mathrm{~s}^{-2}$; green curves in Fig. 8c]. The third profile flips the lowest $6 \mathrm{~km}$ and the $6 \mathrm{~km}$ directly above in the original sounding $\left[N^{2}(6.5 \mathrm{~km})=1.07 \times 10^{-4} \mathrm{~s}^{-2}\right.$; red curves in Fig. 8c], resulting in a weakly stratified lower level and a more strongly stratified level directly above.

Figure $8 \mathrm{c}$ shows the results of the simulations with the solid curves depicting the $\mathrm{LH}+\mathrm{LC}$ experiments and the dashed lines the corresponding LH-only runs. Again, the simulations with LC included yielded stronger lower-level tangential wind accelerations. Moreover, the role of static stability is similar to that of inertial stability but in the vertical direction. Higher values of $N^{2}$ lead to a reduction of the vertical winds and the secondary circulation, which in turn weakens the radial inflow and the advection of absolute vorticity. Specifically, the maximum updraft speeds located at $6.5 \mathrm{~km}$ were $0.24,0.47$, and $0.62 \mathrm{~m} \mathrm{~s}^{-1}$ for the doubled, constant, and flipped sounding profiles, respectively. The corresponding maximum inward pointing radial winds at the lower level were $-0.59,-1.17$, and $-1.55 \mathrm{~m} \mathrm{~s}^{-1}$, respectively. These differences in the secondary circulation cause a reduction in the inward transport of absolute vorticity and, therefore, a weaker low-level tangential wind acceleration in the case with large $N^{2}$.

\section{Discussion and conclusions}

Latent heat release from condensational heating has been recognized as one of the dominating energy sources of a tropical cyclone. In this paper, we examined other microphysical processes which may also play an important role. From an analysis of a real-case simulation of Hurricane Katrina (2005), it was found that cooling from evaporation and melting of some frozen hydrometeors radially outside the eyewall region can have similar magnitudes as condensational heating. Based on this finding, idealized thermally forced experiments were performed. The specified heating and cooling functions mimic those found in the Hurricane Katrina run. The results indicated that the addition of cooling enhances the lower-level inward radial winds,

\footnotetext{
${ }^{1}$ Parameter $N^{2}(6.5 \mathrm{~km})$ was chosen to compare the three profiles because $6.5 \mathrm{~km}$ is the approximate height of the maximum vertical winds in the three static stability runs.
}

which in turn increases the acceleration of the lowerlevel tangential winds through an enhanced transport of absolute vorticity. Sensitivity experiments on varying the structure of the cooling functions and the background state of the vortex demonstrate that the lowerlevel tangential wind acceleration is more sensitive to changes in the vertical structure and location of the cooling than the radial characteristics. In addition, the lower-level acceleration is sensitive to variations in the inertial and static stabilities rather than the vertical tangential wind shear of the initial vortex and its environment. Sensitivity tests to the maximum magnitude of cooling were performed but not shown here. It was found that the stronger the magnitude of cooling, the larger the spin of the lower-level tangential winds due to an enhancement of the inward-pointing radial winds.

From the results presented, a physical picture as to how ice hydrometeors influence low-level wind acceleration emerges. Principally, snow and graupel in a tilted eyewall falls to the melting level radially outward of the region of condensational heating. The melting ice hydrometeors affect cooling around the melting level through the latent heat of fusion. The melted snow and graupel form rainwater, which precipitates to the unsaturated levels below and affects cooling through the latent heat of vaporization. Consequently, a larger radial gradient of heating and an enhanced low-level cooling are realized to affect the acceleration of the low-level tangential wind.

Figure 9 illustrates schematically how low-level cooling (LC) may enhance the lower-level radial transport of absolute vorticity. There is an inward-pointing radial branch at the lowest levels, which turns upright in the eyewall region. A downdraft exists within the cooling region and, on reaching the lower levels, it enhances the radially inward pointing branch of the secondary circulation but tends to prevent the increase of tangential velocity just outside of the radius of the maximum of LC. The enhancement of the radial winds results in an increase in the inward transport of absolute vorticity and accelerates the lower-level tangential winds. It should be noted that this secondary coupling must be significantly smaller than the initial inward, upward, outward circulation from the lowlevel heating (LH; red arrows in Fig. 9). The theoretical maximum amount of cooling from either melting or evaporation of hydrometeors is equal to the total amount of water vapor condensing. Since not all of the condensate precipitates out, this implies that cooling from evaporation and melting must be smaller than the total heating in the eyewall region. Since condensation is always present in the eyewall, the cooling outside does not create the secondary circulation but acts only to enhance it (blue arrows in Fig. 9). Some quantification of this cooling has been documented by Didlake and Houze (2013); however, their 


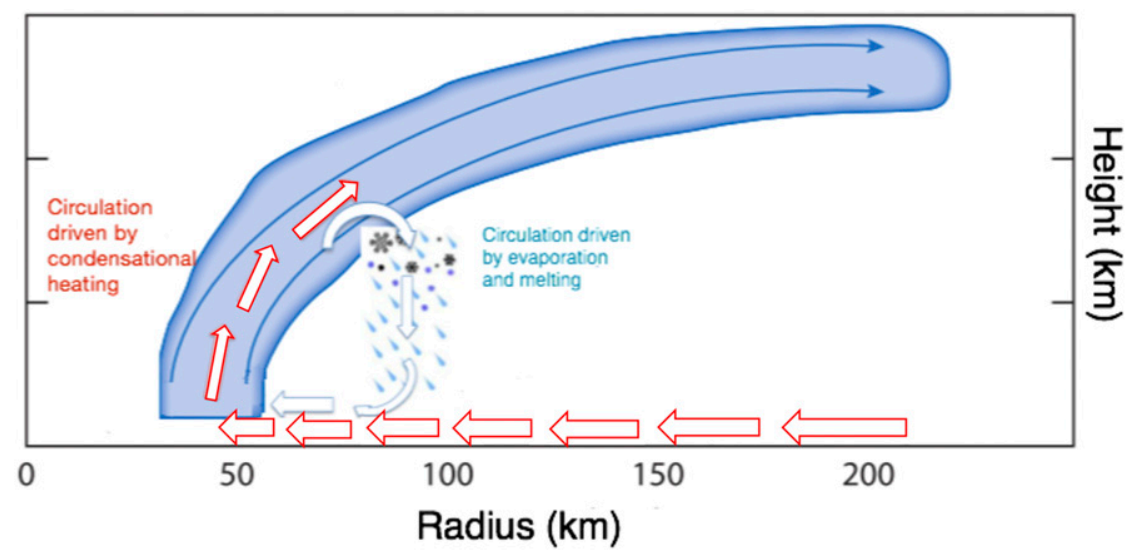

FIG. 9. Schematic of how low-level cooling outside the main eyewall region can influence the secondary circulation, and thus the tangential wind acceleration, of a tropical cyclone. The red arrows represent the circulation driven by condensational heating where there exists an inwardpointing radial wind branch near the surface, which turns vertically upward in the eyewall region. The blue arrows represent the induced circulation from the cooling components occurring radially outside of the eyewall region. Here there exists an enhanced downdraft, which near the surface adds strength to the inward-pointing radial winds from the condensational driven circulation to create a stronger inward response.

focus was on the rainbands of a tropical cyclone as opposed to the main eyewall region. Further experiments and observational data are required in order to more accurately quantify how much the cooling influences the secondary circulation.

Heating vertically higher in the atmosphere, arising from the deposition of snow, may play a key role in tropical cyclone dynamics. Chan and Chan (2016) found that this stronger heating toward the top of the TC resulted in a stronger secondary circulation and a more intense storm. The additional heating higher may act to transport larger values of tangential momentum vertically (Chan and Chan 2016). Depending on the location of the majority of the deposition of snow, this could impact how strong the vertical tangential wind shear is in the eyewall region, the slope of the radius of maximum winds (RMW) with height, and the strength of the secondary circulation. There is an ongoing debate as to whether the slope of the RMW with height may be related to the intensity of a storm. Some papers have demonstrated such a relationship (e.g., Shea and Gray 1973; Dodge et al. 1999), while other studies have shown no relationship (e.g., Marks and Houze 1987; Stern and Nolan 2009). These ideas will be tested and expanded on in a future publication.

It should be stated that there are many caveats to using the idealized methodology presented in this paper. As mentioned, the main goal of this study is to gain a better understanding of how the diabatic heating/cooling arising from microphysical (MP) processes impact(s) a tropical cyclone (TC). However, many possible complications were ignored in these simulations. For example, the addition of a planetary boundary layer would significantly alter the radial wind structure and thus the transport of absolute vorticity. Another neglected aspect is the role that ice-phase processes may play in influencing the temperature and moisture structures through cloud radiative feedback. It has been well documented that this feedback impacts both the track and strength of a tropical cyclone (Fovell et al. 2009, 2010), the widening of the eye, and the enhancement of the secondary circulation (Bu et al. 2014; Fovell et al. 2016). Thus, future research must take into account both the influences from the planetary boundary layer and cloudradiative feedback processes to further our understanding of the internal dynamics of a tropical cyclone.

Acknowledgments. The authors thank the three anonymous reviewers for their valuable suggestions. The research reported here is supported by the NSERC/ Hydro-Quebec Industrial Research Chair Program (IRCPJ/381215-14).

\section{APPENDIX}

\section{Simulations Using the Sawyer-Eliassen Equation}

To further support the idea that the cooling outside of the RMW boosts the heating inside to enhance the secondary circulation, experiments using the Sawyer-Eliassen (SE) model were performed. The SE equation for the streamfunction [Eq. (14) of Bui et al. (2009) excluding the force applied to the air in the azimuthal direction] and the form of the thermal forcing used are 

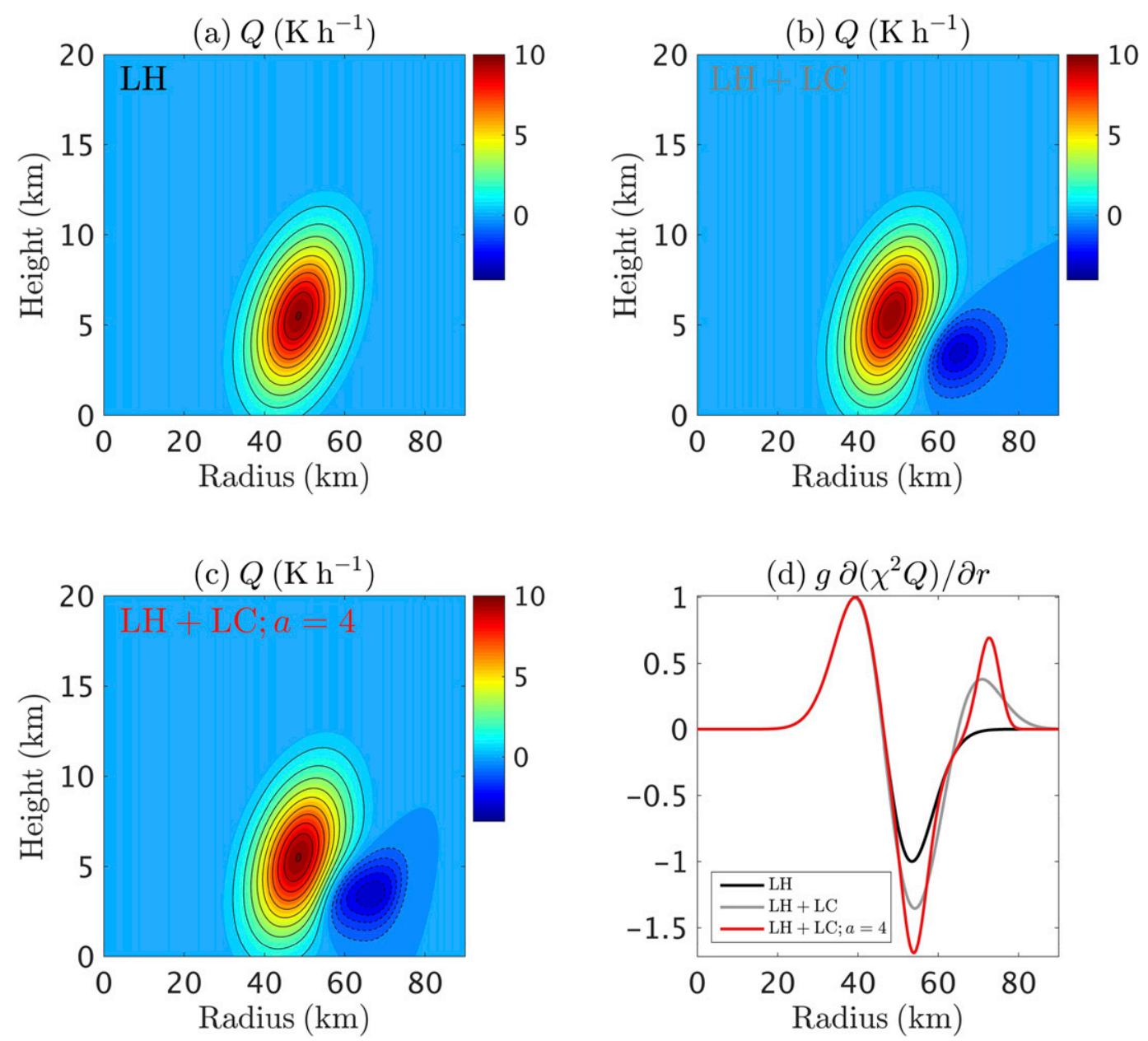

FIG. A1. (a)-(c) Radius-height cross sections of the prescribed diabatic heating/cooling rate $Q\left(\mathrm{~K} \mathrm{~h}^{-1}\right)$ used to drive the SE equation: (a) diabatic heating rate only $(\mathrm{LH})$, (b) diabatic heating rate plus low-level cooling ( $\mathrm{LH}+\mathrm{LC})$, and (c) diabatic heating and cooling rates. Here, the cooling rate decays more sharply (relative to the previous case) in the radial direction ( $\mathrm{LH}+\mathrm{LC} ; \alpha=4)$. (d) Radial profile of the first forcing term on the RHS of Eq. (A1) (SE equation). The profiles are extracted at $z=3.4 \mathrm{~km}$ and are normalized to their respective maximum positive value.

$$
\frac{\partial}{\partial r}\left[-g \frac{\partial \chi}{\partial z} \frac{1}{r \rho} \frac{\partial \psi}{\partial r}-\frac{\partial(\chi C)}{\partial z} \frac{1}{r \rho} \frac{\partial \psi}{\partial z}\right]+\frac{\partial}{\partial z}\left\{\left[\xi \chi(\zeta+f)+C \frac{\partial \chi}{\partial r}\right] \frac{1}{r \rho} \frac{\partial \psi}{\partial z}-\frac{\partial(\chi C)}{\partial z} \frac{1}{r \rho} \frac{\partial \psi}{\partial r}\right\}=g \frac{\partial}{\partial r}\left(\chi^{2} Q\right)+\frac{\partial}{\partial z}\left(C \chi^{2} Q\right),
$$

$$
Q(r, z)=Q_{\max / \min } \exp \left[-\frac{\left(r-r_{c}-\gamma z\right)^{\alpha}}{\delta_{r}^{\alpha}}-\frac{\left(z-z_{c}\right)^{\beta}}{\delta_{z}^{\beta}}\right]
$$

Here, $r$ is radius; $z$ is height; $\chi \equiv 1 / \theta$, in which $\theta$ is the potential temperature; $\rho$ is density; $\zeta=\partial(r v) / r \partial r$ is the vertical component of relative vorticity; $f$ is the Coriolis parameter; $g$ is the gravitational acceleration; $C=f v+v^{2} / r$ is the sum of the Coriolis and centrifugal terms; $\xi=2 v / r+f$ is twice the local absolute angular velocity; $Q$ is the diabatic heating/cooling rate; $Q_{\max } / \min$ is the maximum amplitude of the heating/cooling rate; $r_{c}\left(z_{c}\right)$ is the center of the heating/ cooling in the radial (vertical) direction; $\delta_{r}\left(\delta_{z}\right)$ is the radial (vertical) half-width; $\alpha(\beta)$ controls the degree of decay in the radial (vertical) direction; and $\gamma$ determines the slope of the heating/cooling with height.

Equation (A1) indicates that the thermal generation of a secondary circulation $\left[u=-(r \rho)^{-1} \partial \psi / \partial z\right.$ and $w=$ $\left.(r \rho)^{-1} \partial \psi / \partial r\right]$ is closely related to the radial and vertical 


$$
\partial v / \partial t\left(\times 10^{-3} \mathrm{~m} \mathrm{~s}^{-2}\right)
$$
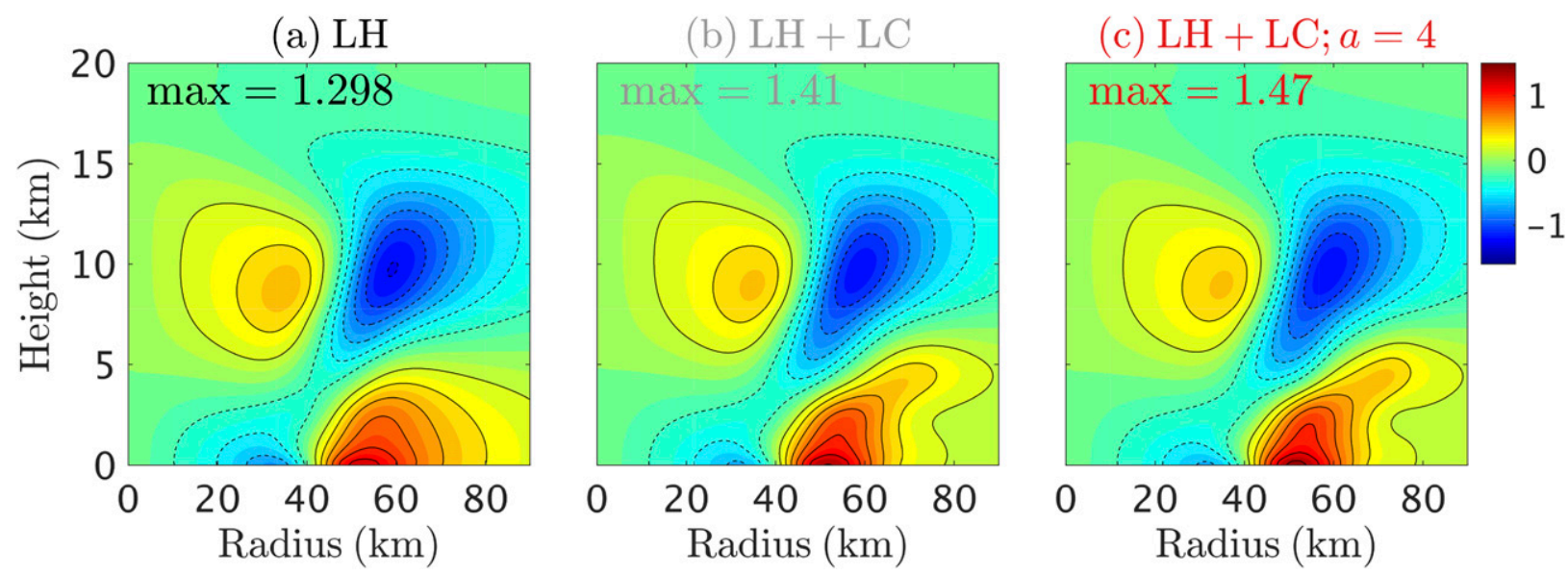

FIG. A2. Radius-height cross sections of the resultant azimuthal wind acceleration $\partial v / \partial t\left(\times 10^{-3} \mathrm{~m} \mathrm{~s}^{-2}\right)$ : (a) LH case, (b) LH $+\mathrm{LC}$ case, and (c) $\mathrm{LH}+\mathrm{LC}$ with $\alpha=4$ case.

gradients of the diabatic heating/cooling rate. To assess whether in this balanced framework the presence of low-level cooling (denoted as LC in the manuscript) leads to a larger acceleration (a consistent finding in the idealized WRF simulations), three experimental cases are examined using the SE model. In the first case, the SE equation is forced only with a heating term (denoted as LH in the manuscript), whereas in the second case both a heating and low-level cooling are taken into consideration $(\mathrm{LH}+\mathrm{LC})$. Figures A1a and $\mathrm{A} 1 \mathrm{~b}$ show the respective specified spatial structures. Note that the parameters $r_{c}, z_{c}, \delta_{r}, \delta_{z}, \alpha, \beta$, and $\gamma$ used in Eq. (A2) to construct these patterns are the same as those listed in
Table 1. In the third case, the SE equation is again forced with both a heating and cooling term, but with the latter experiencing this time a sharper radial decay (controlled by the parameter $\alpha$, where $\alpha=4$ is depicted in Fig. A1c). It is worth remarking that the addition of even weak cooling can result in significant differences in the magnitude of the forcing terms [especially the one related to the radial gradient of $Q$; first term on the RHS of Eq. (A1)]. This is clearly depicted in Fig. A1d, which compares the radial profiles of the aforementioned forcing term for the three cases.

Figure A2 depicts the spatial structure $(r-z)$ along with the maximum values of the azimuthal wind acceleration
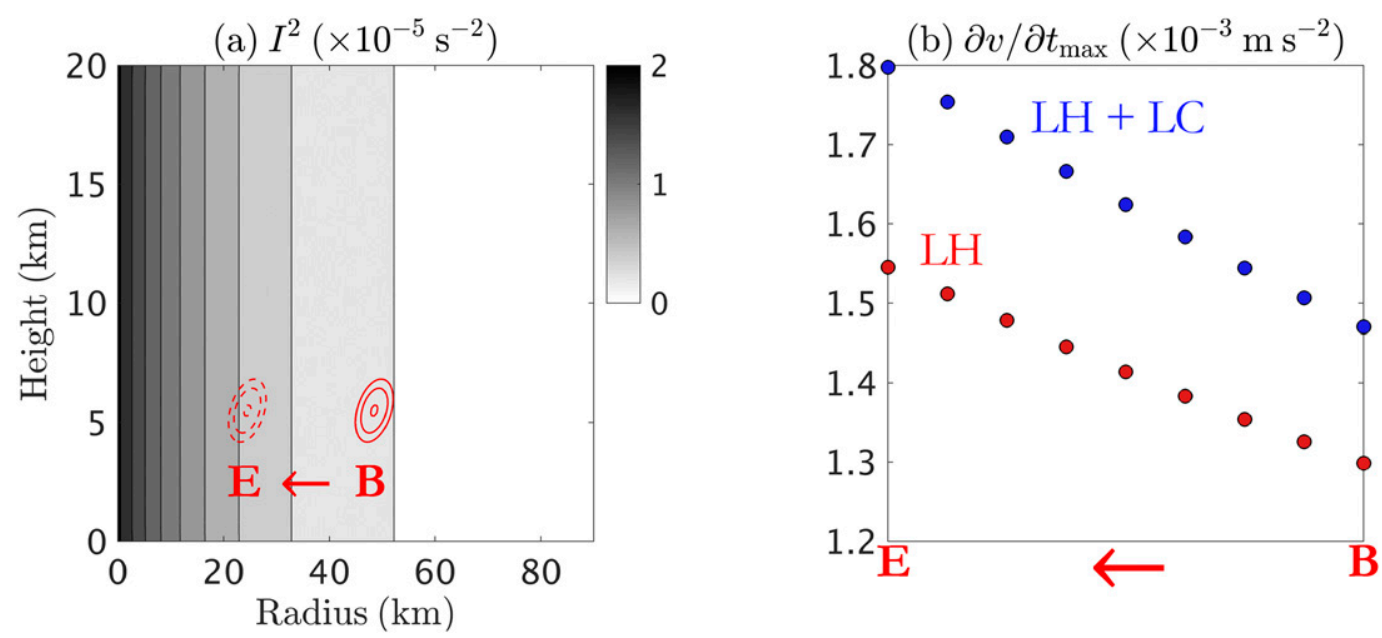

FIG. A3. (a) Radius-height cross section of inertial stability (gray shading represents the values of $I^{2}$ ). The solid (dashed) red contours traces the initial B (final E) position of the diabatic heating-rate core; the illustrated $Q$ contours are 8,9 , and $10 \mathrm{~K} \mathrm{~h}^{-1}$. (b) Maximum azimuthal wind acceleration vs radial location of the thermal forcing (from $\mathrm{B}$ to $\mathrm{E}$ implies an inward radial shift) for diabatic heating only (LH; red dots) and diabatic heating plus cooling (LH + LC; blue dots). 
$\partial v / \partial t$. Note that in the present scenario (axisymmetric barotropic vortex, absence of friction, absence of eddy fluxes, and absence of subgrid-scale diffusion), $\partial v / \partial t$ reduces to

$$
\frac{\partial v}{\partial t}=-u(\zeta+f) .
$$

Here, there are a few things to note. First, the resultant spatial patterns of $\partial v / \partial t$ are qualitatively similar to those extracted from the idealized WRF simulations (cf. Fig. A2 with Fig. 6 showing the computed $\partial v / \partial t$ at $180 \mathrm{~min}$ of the simulation). There are some quantitative differences due largely to the fact that the vertical advection of azimuthal momentum is zero in the current SE framework but is nonzero in the prognostic WRF framework at $180 \mathrm{~min}$. Second, the largest acceleration occurs in the presence of cooling. This finding is consistent with what was found from the WRF experiment and provides further confidence that the consideration of relatively weak low-level cooling can indeed induce nontrivial changes in the low-level wind acceleration.

An additional important factor that can determine the strength and overall structure of the transverse circulation in the SE model is the inertial stability at the forcing location. To determine whether the above outcome (low-level wind acceleration becomes larger in the presence of cooling) remains valid if the inertial stability at the forcing location is changed, two new sets of SE calculations are performed. In the first set, the thermal forcing comprising only of diabatic heating (LH) is gradually shifted radially inward toward values of higher inertial stability. In the second set, the same radial shift is repeated, but this time the thermal forcing comprises both heating and cooling ( $\mathrm{LH}+\mathrm{LC})$. For illustrative purposes, Fig. A3a sketches the initial (denoted by the red capital letter B) and final (denoted by the red capital letter E) radial location of the LH core (red contours) superimposed on the basic-state inertial stability $I^{2}$. Figure A3b illustrates the resultant maximum acceleration for the two cases. Focusing first on the LH case, it can be seen that as the thermal source shifts to regions with higher values of $I^{2}$ (from $\mathrm{B}$ to $\mathrm{E}$ ), the maximum acceleration becomes larger. This is, of course, an expected outcome that has been previously documented in the literature. For the $\mathrm{LH}+\mathrm{LC}$ experiment, it is evident that the presence of cooling results in a systematic (nontrivial) amplification of the maximum acceleration. This result casts little doubt about the significant boosting contribution of low-level cooling in the acceleration of the low-level winds.

\section{REFERENCES}

Braun, S., 2002: A cloud-resolving simulation of Hurricane Bob (1991): Storm structure and eyewall buoyancy. Mon. Wea. Rev., 130, 1573-1592, https://doi.org/10.1175/1520-0493(2002) $130<1573$ :ACRSOH $>2.0 . \mathrm{CO} ; 2$.
Bu, Y. P., R. G. Fovell, and K. L. Corbosiero, 2014: Influence of cloud-radiative forcing on tropical cyclone structure. J. Atmos. Sci., 71, 1644-1662, https://doi.org/10.1175/JAS-D-13-0265.1.

Bui, H., R. K. Smith, M. T. Montgomery, and J. Peng, 2009: Balanced and unbalanced aspects of tropical cyclone intensification. Quart. J. Roy. Meteor. Soc., 135, 1715-1731, https://doi.org/10.1002/qj.502.

Chan, K. T. F., and J. C. L. Chan, 2016: Sensitivity of the simulation of tropical cyclone size to microphysics schemes. Adv. Atmos. Sci., 33, 1024-1035, https://doi.org/10.1007/s00376-016-5183-2.

Chou, M.-D., and M. J. Suarez, 1999: A solar radiation parameterization for atmospheric studies. Tech. Memo. NASA/TM-1999-104606, Vol. 15, 38 pp., http://gmao.gsfc.nasa.gov/pubs/docs/Chou136.pdf.

Davis, C., and Coauthors, 2008: Prediction of landfalling hurricanes with the advanced hurricane WRF Model. Mon. Wea. Rev., 136, 1990-2005, https://doi.org/10.1175/2007MWR2085.1.

DeMaria, M., and M. Mainelli, 2005: Further improvements to the Statistical Hurricane Intensity Prediction Scheme (SHIPS). Wea. Forecasting, 20, 531-543, https://doi.org/10.1175/WAF862.1.

Didlake, A. C., Jr., and R. A. Houze Jr., 2013: Dynamics of the stratiform sector of a tropical cyclone rainband. J. Atmos. Sci., 70, 1891-1911, https://doi.org/10.1175/JAS-D-12-0245.1.

Dodge, P., R. W. Burpee, and F. D. Marks, 1999: The kinematic structure of a hurricane with sea level pressure less than $900 \mathrm{mb}$. Mon. Wea. Rev., 127, 987-1004, https://doi.org/ 10.1175/1520-0493(1999)127<0987:TKSOAH >2.0.CO;2.

Fovell, R. G., and H. Su, 2007: Impact of cloud microphysics on hurricane track forecasts. Geophys. Res. Lett., 34, L24810, https://doi.org/10.1029/2007GL031723.

— K. K. Corbosiero, and H.-C. Kuo, 2009: Cloud microphysics impact on hurricane track as revealed in idealized experiments. J. Atmos. Sci., 66, 1764-1778, https://doi.org/10.1175/2008JAS2874.1.

,-- A. Seifert, and K.-N. Liou, 2010: Impact of cloudradiative processes on hurricane track. Geophys. Res. Lett., 37, L07808, https://doi.org/10.1029/2010GL042691.

, Y. P. Bu, K. L. Corbosiero, W.-W. Tung, Y. Cao, H.-C. Kuo, L.- H. Hsu, and H. Su, 2016: Influence of cloud microphysics and radiation on tropical cyclone structure and motion. Multiscale Convection-Coupled Systems in the Tropics: A Tribute to Dr. Michio Yanai, Meteor. Monogr., No. 56, Amer. Meteor. Soc., https://doi.org/10.1175/AMSMONOGRAPHS-D-15-0006.1.

Grell, G. A., and D. Dévényi, 2002: A generalized approach to parameterizing convection combining ensemble and data assimilation techniques. Geophys. Res. Lett., 29, 1693, https:// doi.org/10.1029/2002GL015311.

Hack, J., and W. H. Schubert, 1986: Nonlinear response of atmospheric vortices to heating by organized cumulus convection. J. Atmos. Sci., 43, 1559-1573, https://doi.org/10.1175/15200469(1986)043<1559:NROAVT>2.0.CO;2.

Heymsfield, G. M., J. B. Halverson, J. Simpson, L. Tian, and T. P. Bui, 2001: ER-2 Doppler radar investigations of the eyewall of Hurricane Bonnie during the Convection and Moisture Experiment3. J. Appl. Meteor. Climatol., 40, 1310-1330, https://doi.org/ 10.1175/1520-0450(2001)040<1310:EDRIOT>2.0.CO;2.

Holland, G., and R. Merrill, 1984: On the dynamics of tropical cyclone structural changes. Quart. J. Roy. Meteor. Soc., 110, 723-745, https://doi.org/10.1002/qj.49711046510.

Iacono, M. J., J. S. Delamere, E. J. Mlawer, M. W. Shephard, S. A. Clough, and W. D. Collins, 2008: Radiative forcing by longlived greenhouse gases: Calculations with the AER radiative transfer models. J. Geophys. Res., 113, D13103, https://doi.org/ 10.1029/2008JD009944.

Janjić, Z. I., 1994: The step-mountain Eta coordinate model: Further developments of the convection, viscous sublayer, 
and turbulence closure schemes. Mon. Wea. Rev., 122, 927-945, https://doi.org/10.1175/1520-0493(1994)122<0927: TSMECM $>2.0 . \mathrm{CO} ; 2$.

- 1996: The surface layer in the NCEP Eta Model. Preprints, 11th Conf. on Numerical Weather Prediction, Norfolk, VA, Amer. Meteor. Soc., 354-355.

- 2002: Nonsingular implementation of the Mellor-Yamada level 2.5 scheme in the NCEP Meso model. NCEP Office Note 437, 61 pp., http://www.emc.ncep.noaa.gov/officenotes/ newernotes/on437.pdf.

Jin, Y., and Coauthors, 2014: The impact of ice phase cloud parameterizations on tropical cyclone prediction. Mon. Wea. Rev., 142, 606-625, https://doi.org/10.1175/MWR-D-13-00058.1.

Jordan, C., 1958: Mean soundings for the West Indies area. J. Meteor., 15, 91-97, https://doi.org/10.1175/1520-0469(1958) 015<0091:MSFTWI >2.0.CO;2.

Knabb, R. D., J. R. Rhome, and D. P. Brown, 2005: Tropical cyclone report: Hurricane Katrina. Tech. Rep., 43 pp., https:// www.nhc.noaa.gov/data/tcr/AL122005_Katrina.pdf.

Lord, S., 1984: Role of a parameterized ice-phase microphysics in an axisymmetric, nonhydrostatic tropical cyclone model. J. Atmos. Sci., 41, 2836-2848, https://doi.org/10.1175/15200469(1984)041<2836:ROAPIP > 2.0.CO;2.

- and J. Lord, 1988: Vertical velocity structure in an axisymmetric, nonhydrostatic tropical cyclone model. J. Atmos. Sci., 45, 1453-1461, https://doi.org/10.1175/1520-0469(1988)045<1453: VVSIAA $>2.0 . \mathrm{CO} ; 2$.

Malkus, J., 1958: On the structure and maintenance of the mature hurricane eye. J. Meteor., 15, 337-349, https://doi.org/10.1175/ 1520-0469(1958)015<0337:OTSAMO > 2.0.CO;2.

- , and H. Riehl, 1960: On the dynamics and energy transformations in steady-state hurricanes. Tellus, 12, 1-20, https:// doi.org/10.3402/tellusa.v12i1.9351.

Marks, F. D., and R. A. Houze Jr., 1987: Inner core structure of Hurricane Alicia from airborne Doppler radar observations. J. Atmos. Sci., 44, 1296-1317, https://doi.org/10.1175/15200469(1987)044<1296:ICSOHA > 2.0.CO;2.

—_ and Coauthors, 1998: Landfalling tropical cyclones: Forecast problems and associated research opportunities. Bull. Amer. Meteor. Soc., 79, 305-323, https://doi.org/10.1175/ 1520-0477(1998)079<0285:QPFROT>2.0.CO;2.

McFarquhar, G. M., H. Zhang, G. Heymsfield, J. B. Halverson, R. Hood, J. Dudhia, and F. Marks, 2006: Factors affecting the evolution of Hurricane Erin (2001) and the distributions of hydrometeors: Role of microphysical processes. J. Atmos. Sci., 63, 127-150, https://doi.org/10.1175/JAS3590.1.

Ohno, T., and M. Satoh, 2015: On the warm core of a tropical cyclone formed near the tropopause. J. Atmos. Sci., 72, 551-571, https://doi.org/10.1175/JAS-D-14-0078.1.

Pattnaik, S., and T. N. Krishnamurti, 2007: Impact of cloud microphysical processes on hurricane intensity. Part 2: Sensitivity experiments. Meteor. Atmos. Phys., 97, 127-147, https://doi.org/ 10.1007/s00703-006-0248-x.

Paull, G., K. Menelaou, and M. K. Yau, 2017: Sensitivity of tropical cyclone intensification to axisymmetric heat sources: The role of inertial stability. J. Atmos. Sci., 74, 2325-2340, https://doi.org/ 10.1175/JAS-D-16-0298.1.

Pendergrass, A. G., and H. E. Willoughby, 2009: Diabatically induced secondary flows in tropical cyclones. Part I: Quasi-steady forcing. Mon. Wea. Rev., 137, 805-821, https://doi.org/10.1175/ 2008MWR2657.1.
Rogers, R., 2010: Convective-scale structure and evolution during a high-resolution simulation of tropical cyclone rapid intensification. J. Atmos. Sci., 67, 44-70, https://doi.org/10.1175/ 2009JAS3122.1.

_ , and Coauthors, 2006: The intensity forecasting experiment: A NOAA multiyear field program for improving tropical cyclone intensity forecasts. Bull. Amer. Meteor. Soc., 87, 15231537, https://doi.org/10.1175/BAMS-87-11-1523.

_- and Coauthors, 2013: NOAA's hurricane intensity forecasting experiment: A progress report. Bull. Amer. Meteor. Soc., 94, 859-882, https://doi.org/10.1175/BAMS-D-12-00089.1.

Rotunno, R., and K. A. Emanuel, 1987: An air-sea interaction theory for tropical cyclones. Part II: Evolutionary study using a nonhydrostatic axisymmetric numerical model. J. Atmos. Sci. 44, 542-561, https://doi.org/10.1175/1520-0469(1987)044<0542: AAITFT $>2.0 . \mathrm{CO} ; 2$.

Sawada, M., and T. Iwasaki, 2007: Impacts of ice phase processes on tropical cyclone development. J. Meteor. Soc. Japan, 85, 479-494, https://doi.org/10.2151/jmsj.85.479.

- , and 2010: Impacts of evaporation from raindrops on tropical cyclones. Part I: Evolution and axisymmetric structure. J. Atmos. Sci., 67, 71-83, https://doi.org/10.1175/2009JAS3040.1.

Shea, D. J., and W. M. Gray, 1973: The hurricane's inner core region. I. Symmetric and asymmetric structure. J. Atmos. Sci., 30, 1544-1564, https://doi.org/10.1175/1520-0469(1973)030<1544: THICRI $>2.0 . \mathrm{CO} ; 2$.

Simpson, J., and J. Halverson, 1998: On the role of "hot towers" in tropical cyclone formation. Meteor. Atmos. Phys., 67, 15-35, https://doi.org/10.1007/BF01277500.

Skamarock, W. C., and Coauthors, 2008: A description of the Advanced Research WRF version 3. NCAR Tech. Note NCAR/ TN-475+STR, 113 pp., https://doi.org/10.5065/D68S4MVH.

Stern, D. P., and D. S. Nolan, 2009: Reexamining the vertical structure of tangential winds in tropical cyclones: Observations and theory. J. Atmos. Sci., 66, 3579-3600, https://doi.org/ 10.1175/2009JAS2916.1

Thompson, G., P. Field, R. M. Rasmussen, and W. D. Hall, 2008: Explicit forecasts of winter precipitation using an improved bulk microphysics scheme. Part II: Implementation of a new snow parameterization. Mon. Wea. Rev., 136, 5095-5115, https://doi.org/10.1175/2008MWR2387.1.

Willoughby, H. E., 1998: Tropical cyclone eye thermodynamics. Mon. Wea. Rev., 126, 3053-3067, https://doi.org/10.1175/15200493(1998)126<3053:TCET > 2.0.CO;2.

- H. Jin, S. Lord, and J. Piotrowicz, 1984: Hurricane structure and evolution as simulated by axisymmetric, non-hydrostatic numerical model. J. Atmos. Sci., 41, 1169-1186, https://doi.org/10.1175/ 1520-0469(1984)041<1169:HSAEAS $>2.0$. CO;2.

Yamasaki, M., 2013: A study on the effects of the ice microphysics on tropical cyclones. Adv. Meteor., 2013, 573786, https:// doi.org/10.1155/2013/573786.

Zhu, T., and D.-L. Zhang, 2006: Numerical simulation of Hurricane Bonnie (1998). Part II: Sensitivity to varying cloud microphysical processes. J. Atmos. Sci., 63, 109-127, https://doi.org/ 10.1175/JAS3599.1.

Zipser, E., 2003: Some views on "hot towers" after 50 years of tropical field programs and two years of TRMM data. Cloud Systems, Hurricanes, and the Tropical Rainfall Measuring Mission (TRMM) - A Tribute To Dr. Joanne Simpson, Meteor. Monogr., No. 51,49-58, https://doi.org/10.1175/0065-9401(2003) 029<0049:CSVOHT>2.0.CO;2. 